\title{
Argentinos en España: una comparación entre la inserción laboral de exiliados y migrantes económicos
}

\section{Argentinians in Spain: a comparison between the employment of exiles and economic migrants}

\author{
Fernando Osvaldo Esteban \\ Universidad de Buenos Aires \\ Consejo Nacional de Investigaciones Científicas y Técnicas
}

(Argentina)

fernandosvaldoesteban@gmail.com

\section{Resumen}

Este artículo analiza la movilidad ocupacional trasnacional de la población argentina en España, desde el último empleo en origen al primero en destino, de tres flujos migratorios con características propias: el exilio político (1975-1983), las primeras migraciones económicas (1984-1999) y las últimas de carácter masivo (2000-2007). El análisis se apoya en la explotación de la Encuesta Nacional de Inmigrantes realizada en España en 2007. Los resultados comprueban que los argentinos no mejoraron la situación laboral que tenían antes de emigrar, por el contrario, experimentaron una movilidad descendente desde una posición más alta en Argentina. Asimismo, se constata una mejor inserción laboral de los flujos migratorios más antiguos, en particular el del exilio, debido a factores de índole estructural como la política migratoria y la estructura del mercado de trabajo y, en menor medida, a factores de índole individual como el nivel educativo, el sexo o la edad de los migrantes.

Palabras Clave: España, inmigración argentina, exilio, mercado de trabajo, movilidad laboral. 


\begin{abstract}
This article analyses the transnational occupational mobility of argentines in Spain, from the last employment in origin to the first in destination. The article focuses on three different migratory flows: political exile (19751983), the first economic migration (1984-1999) and the last one (2000-2007). The National Immigrant Survey conducted in Spain in 2007 provided the statistical data. The results proved that immigrants did not improve the employment status they had before emigrating; on the contrary, they experienced downward mobility from a higher position in Argentina. In addition, we found a better employment of older migration flows, specially exiles, due to structural factors such as migration policy and the structure of the labor market and, to a lesser extent, individual factors such as educational level, sex or age of migrants.
\end{abstract}

Key Words: Spain, Argentine immigration, Labor market, Labor mobility, Exile

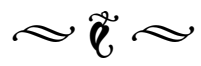

\section{Introducción}

La emigración de argentinos a España regresó a las agendas de los científicos sociales en las últimas décadas. A final de los años ` 90 comenzaron a desarrollarse investigaciones sobre el exilio político, principalmente en el ámbito de los estudios de la historia reciente. Las investigaciones abarcaron diversos aspectos del desplazamiento. Entre éstos destacan la huida del país, la militancia política, las modalidades represivas y los tipos de exilio, la (re)construcción de identidades, la denuncia de la dictadura, ${ }^{1}$ el asociacionismo y la lucha por la defensa de los derechos humanos, ${ }^{2}$ los juicios de

1. JENSEN, Silvina, La provincia flotante. El exilio argentino en Cataluña (1976-2006), Casa América, Barcelona, 2008.

2. MIRA DELLI-ZOTTI, Guillermo, “¿Sobrevivir o vivir en Madrid? Exiliados argentinos del '76”, en ESPINA BARRIO, Ángel (editor) Antropología en Castilla y León e Iberoamérica V. Emigración e integración cultural, Universidad de Salamanca, Salamanca, 2003, pp. 187-198; MIRA DELLI-ZOTTI, Guillermo, ESTEBAN, Fernando, "El flujo que no cesa. Aproximación a las razones, cronología y perfil de los argentinos radicados en España (1975-2001)", en
Madrid, ${ }^{3}$ los debates políticos y sus representaciones en la prensa y el cine, ${ }^{4}$ las patologías psicológicas de las víctimas. ${ }^{5}$ En la actualidad, las investigaciones profundizan dimensiones regionales, comparadas y transnacionales en el análisis de retornos, militancias humanitarias o lógicas represivas. ${ }^{6}$ Sin embargo, la inserción socioeconómica en destino, particularmente, los aspectos relacionados con el empleo, no llamó la atención de los investigadores o sólo de un modo tangencial.

Historia Actual On-Line, Cádiz, 2003, № 2, pp. 31-43; JENSEN, Silvina, La provincia flotante... Op. Cit.

3. MÁS, Fernando, De Nüremberg a Madrid. Historia intima de un juicio, Grijalbo, Buenos Aires, 1999.

4. ACTIS, Walter; ESTEBAN, Fernando Osvaldo, "Argentinos en España: inmigrantes a pesar de todo", en Migraciones, Madrid, 2008, No 23, pp. 79-115.

5. GRINBERG, León y GRINBERG, Rebeca, Psicoanálisis de la emigración y del exilio, Alianza, Madrid, 1982.

6. JENSEN, Silvina, LASTRA, Soledad, "El problema de las escalas en el campo de estudio de los exilios políticos argentinos recientes", en Avances del Cesor, Rosario, 2015, V.XII, pp. 97-119. 
Posteriormente, las investigaciones se enfocaron sobre la emigración masiva que tuvo lugar en Argentina a consecuencia de la crisis de 2001. En esta oportunidad, muchos estudios indagaron la inserción laboral en destino. ${ }^{7}$ En términos generales, coincidieron en que la incorporación al mercado laboral se concentró en el segmento secundario y no satisfacía las expectativas iniciales de los migrantes. Resaltaron también que los argentinos presentaron una inserción más diversificada que otros colectivos de inmigrantes extracomunitarios y que incluía actividades de mayor prestigio social.

La mayor parte de las investigaciones utilizaron una metodología de corte cualitativo, con lo cual, sus conclusiones no pueden extenderse al conjunto del colectivo. Sin embargo, a partir de la publicación de la Encuesta Nacional de Inmigrantes (en adelante, ENI) realizada por el Instituto Nacional de Estadística de España (en adelante, INE) en 2007, algunos estudios explotaron datos estadísticos a nivel nacional y describieron exhaustivamente los principales aspectos de la inserción laboral de los argentinos con representatividad estadística. ${ }^{8}$ Así, se

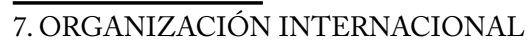
PARA LAS MIGRACIONES (OIM), "Migrantes sudamericanos en España: panorama y políticas", Cuadernos Migratorios, Buenos Aires, Argentina, 2001, No 1, pp. 11-121; JIMÉNEZ, Cecilia, Desclasamiento y reconversiones en las trayectorias de los migrantes argentinos de clases medias, Tesis Doctoral, Universidad Complutense de Madrid, 2011; GANDINI, Luciana, $\dot{\dot{b}}$ Escapando de la crisis? Un estudio comparativo de trayectorias laborales de migrantes argentinos en la Ciudad de México y Madrid, UNAM-CRIM, Cuernavaca, México, 2015; CACOPARDO, María Cristina, MAGUID, Alicia, MARTÍNEZ, Rosana, "La nueva emigración de argentinos a España: el caso de los argentinos desde una perspectiva comparada", en Papeles de Población, TolucaMéxico, 2007, № 51, pp. 9-44.

8. ESTEBAN, Fernando Osvaldo, Cuatro décadas de pudieron confirmar los hallazgos previos y se avanzó en el análisis de algunos factores determinantes de la inserción económica como el sexo, la edad, la ciudadanía y el nivel de estudios.

Este artículo, a partir de la misma fuente, profundiza sobre el tema mediante un análisis comparativo de la movilidad ocupacional trasnacional desde el último empleo en Argentina al primero en España en tres flujos migratorios con características propias: el exilio (19731983), las primeras migraciones económicas (1984-1999) y el "flujo del corralito" (20002007).

E1 texto está compuesto por los siguientes apartados. En primer lugar, presentamos un breve "estado del arte" sobre los enfoques teóricos que explican la inserción laboral de los inmigrantes en las sociedades receptoras. Luego describimos los diferentes flujos migratorios de argentinos hacia España y sus respectivos contextos de recepción. En segundo término, detallamos las fuentes y la metodología de la investigación. Posteriormente, avanzamos en el análisis de los datos, primero con atención al

migraciones de argentinos a España (1970-2010), Editorial Teseo, Buenos Aires, 2015; ESTEBAN, Fernando Osvaldo, "La inserción laboral de los inmigrantes argentinos en España. Un análisis a partir de la Encuesta Nacional de Inmigrantes 2007", en Argumentos. Revista de Crítica Social, Buenos Aires, 2013, o 15, pp. 285-313; AYSA-LASTRA, María y CACHÓN, Lorenzo, "Movilidad ocupacional segmentada: el caso de los inmigrantes no comunitarios en España”, en Revista Española de Investigaciones Sociológicas, Madrid, 2013, No 144, pp. 23-47; ORGANIZACIÓN INTERNACIONAL PARA LAS MIGRACIONES (OIM), "Migrantes sudamericanos en España...”, Op. Cit; VEIRA, Alberto, STANEK, Mikolaj y CACHON, Lorenzo, "Los determinantes de la concentración étnica en el mercado laboral español", en Revista Internacional de Sociología, Córdoba, 2011, Monográfico No 1, pp. 219-242. 
último empleo en Argentina y luego respecto al primero en España. Por último, se presentan las conclusiones.

\section{Estado de la cuestión}

\section{Los enfoques teóricos}

La literatura sobre la integración de los inmigrantes en el mercado de trabajo se ha centrado más en la asimilación de los inmigrantes y las diferencias entre éstos y los nativos en varios resultados del mercado laboral y menos en su movilidad ocupacional. Esto se debe a que el estudio de la movilidad laboral requiere de datos longitudinales que no siempre están disponibles para los investigadores.

La mayoría de las investigaciones sobre la movilidad ocupacional de los inmigrantes han encontrado un patrón en forma de "U". Esto supone que la inserción laboral inicial se produce en categorías inferiores a las que tenían en el país de origen pero, a medida que aumen$\tan$ la antigüedad en el mercado de trabajo y adquieren competencias, tienen la posibilidad de escalar posiciones hasta volver a la categoría ocupacional que tenían antes de la emigración. La literatura relaciona uno y otro movimiento de manera que cuanto más pronunciada es la disminución, en promedio, más marcado es el aumento subsiguiente. ${ }^{9}$ En este apartado se re-

9. CHISWICK, Barry R., "A longitudinal analysis of the occupational mobility of immigrants", en DENNIS, Barbara (editora) Proceedings of the 30th annual winter meeting, Industrial Relations Research Association, Madison, 1977, pp. 20-27; CHISWICK, Barry, "The effect of Americanization on the Earnings of Foreignborn Men", en The Journal of Political Economy, Chicago, 1978, V. 86, No 5, pp. 897-921; BAUER, Thomas y ZIMMERMANN, Klaus F., "Occupational mobility of ethnic migrants", en IZA Discussion Paper, Bonn, 1999, visan los tres principales mecanismos propuestos en la literatura para explicar estos patrones de movilidad ocupacional de la población inmigrante.

\section{La teoria del capital humano y la transferencia de competencias}

La idea elemental de la teoría del capital humano (en adelante, $\mathrm{TCH}$ ) es que las personas gastan en sí mismas de diversas formas y no sólo para obtener satisfacciones actuales, sino también para obtener ingresos futuros, pecuniarios y no pecuniarios (por ejemplo, en salud, educación, búsqueda de información, formación profesional). ${ }^{10}$ Estos gastos pueden considerarse una inversión más que un consumo y tienen en común que quien decide realizarlos lo hace con un interés futuro ( $\mathrm{y}$ renunciando a recompensas en el presente). ${ }^{11}$ Desde este punto de vista, se entiende que los trabajadores acuden al mercado de trabajo con competencias distintas que responden no sólo a sus capacidades innatas, sino también, y principalmente, a inversiones diferentes en capital humano.

La TCH entiende la estratificación en el mercado de trabajo como resultado de un proceso de ajuste entre la oferta de mano de obra de determinadas características y la demanda de los empleadores. En el caso de trabajadores

No 58, pp. 1-24.

10. BECKER, Gary, "Investment in Human Capital: a Theoretical Analysis", en The Journal of Political Economy, Chicago, 1962, V. 70, № 5, p. 49.

11. BLAUG, Mark, "The Empirical Status of Human Capital Theory: A Slightly Jaundiced Survey", en Journal of Economic Literature, Pittsburgh, 1976, No 14, V. III, pp. 829. 
de origen extranjero, las inserciones laborales diferenciadas en las sociedades receptoras obedecen a las diferencias de productividad y a las desigualdades en las dotaciones de capital humano. Niveles educativos bajos, desconocimiento del idioma local o una limitada experiencia laboral constituyen desventajas que ubican a algunos inmigrantes en las posiciones más bajas de la escala ocupacional. ${ }^{12}$

Además, es habitual que los inmigrantes de primera generación no puedan transferir directamente al "nuevo" mercado de trabajo las competencias que adquirieron en sus países de origen. En otras palabras, suelen tener dificultades para transformar el nivel de instrucción en capital humano. ${ }^{13}$ En cuanto a la transferibilidad de habilidades y niveles educativos, hay que considerar varios aspectos. En primer lugar, los niveles de educación superior son más difíciles de transferir. Por lo tanto, la depreciación entre el último puesto ocupacional en el origen y el primero en destino será mayor para los niveles educativos superiores, aunque luego experimentarán una movilidad laboral más rápida una vez que se establezcan en el país anfitrión. ${ }^{14}$ En segundo término, el patrón "U"

12. CHISWICK, Barry, "A Longitudinal Analysis...", Op. Cit.

13. FRIEDBERG, Rachel, "You Can't Take It with You? Immigrant Assimilation and the Portability of Human Capital", en Journal of Labor Economics, Chicago, 2000, No 18, V. II, pp.221-251; HEATH, Anthony, CHEUNG, Sin Yi, "The Comparative Study of Ethnic Minority Disadvantage", en HEATH, Anthony y CHEUNG, Sin Yi (editores) Unequal chances. Ethnic Minorities in Western Labour Markets, Oxford University Press, Oxford, 2007, pp. 1-44.

14. CHISWICK, Barry, "A Longitudinal Analysis..., Op. Cit.; DULEEP, Harriet O. y REGETS, Mark C., "Immigrants and human-capital investment", en American Economic Review, Nashville, 1999, V. 89, No 2, pp. 186-191. será menos pronunciado para los migrantes de países similares, por ejemplo, entre países desarrollados. En tercer lugar, el tiempo de residencia en la sociedad de acogida aumenta el capital humano de los inmigrantes específicamente adaptado a las necesidades del mercado de trabajo local. ${ }^{15}$

\section{Contexto de recepción: mercado de trabajo, redes sociales y política migratoria}

La perspectiva sociológica ha enfatizado la importancia del contexto de recepción para comprender la integración laboral de los inmigrantes. ${ }^{16} \mathrm{El}$ contexto de recepción cuestiona la existencia de una secuencia única de asimilación de los inmigrantes a la sociedad receptora y propone, en cambio, una diversidad de patrones de asentamiento que dependen de los mercados de trabajo, la política migratoria (control de flujos y servicios sociales) y la influencia de la comunidad y las redes étnicas. Así, los contextos de recepción tienden a constituir modelos más o menos coherentes de

15. CHISWICK, Barry. R., COHEN, Yinon y ZACH, Tzippi, "The labor market status of immigrants: Effects of the unemployment rate at arrival and duration of residence", en Industrial \& Labour Relations Review, Ithaca, 1997, V. 50, № 2, pp. 289-303; LAM, Kit-Chun y LIU, Pak-Wai, "Earnings divergence of immigrants", en Journal of Labor Economics, 2002, 20(1), 86-104.

WEISS, Yoram, SAUER, Robert M., GOTLIBOVSKI, Menachem. "Immigration, search, and loss of skill", en Journal of Labour Economics, Chicago, 2003, V. 21, № 3, 557-591.

16. PORTES, Alejandro y BOROCZ, József, "Contemporary immigration: Theoretical perspectives on its determinants and modes of incorporation", en International Migration Review, Staten Island, 1989, V. 23, No 3, pp. 606-630. 
organización para encauzar las oportunidades vitales de los recién llegados.

En los años setenta surgieron críticas a la teoría del capital humano. La más antigua de éstas proviene de la teoría de la segmentación del mercado de trabajo. ${ }^{17} \mathrm{La}$ idea básica de este enfoque sostiene que el mercado de trabajo está dividido en dos segmentos esencialmente distintos y no competitivos entre sí, denominados el segmento primario y el secundario. ${ }^{18} \mathrm{El}$ primero ofrece puestos de trabajo con salarios relativamente elevados, buenas condiciones de trabajo, posibilidades de promoción, equidad y procedimientos establecidos en cuanto a la administración de leyes laborales y, por encima de todo, estabilidad en el empleo. En cambio, los puestos del sector secundario tienden a estar peor pagados, a tener condiciones de trabajo más desfavorables, pocas posibilidades de promoción y están caracterizados por una considerable inestabilidad de empleo y una elevada rotación. ${ }^{19}$

17. Por ejemplo, las distintas versiones de la teoría institucionalista que resaltan la acción colectiva para mejorar las condiciones de trabajo, el enfoque marxista clásico que distingue tres tipos de población activa (fluctuante, latente y estancada) y los enfoques neomarxistas que ven en la segmentación una estrategia de la patronal para dividir una potencial clase obrera.

18. La propuesta inicial fue formulada por Doeringer y Piore y aplicada luego por este último al caso de la incorporación de trabajadores extranjeros en la economía de los Estados Unidos. Al respecto ver: DOERINGER, Peter y PIORE Michael, Internal labor markets and manpower adjustment, DC Heath and Company, New York, 1971; PIORE, Michael, Birds of passage: Migrant labor and industrial societies, Cambridge University Press, Cambridge, 1979.

19. PIORE, Michael, "Notas para una teoría de la estratificación del mercado de trabajo”, en TOHARIA, Luis, El mercado de trabajo: teorías y aplicaciones. Lecturas seleccionadas, Alianza, Madrid, 1983, pp. 195-221
Desde esta perspectiva, la inmigración extranjera hacia las economías avanzadas se produce por el reclutamiento de mano de obra extranjera para ocupar puestos de trabajo en el segmento secundario. ${ }^{20}$ Ello se debe, básicamente, a dos razones. En primer lugar, porque es habitual que los trabajadores autóctonos rechacen esos empleos y, al mismo tiempo, es difícil seducirlos a través de mejoras en las condiciones de trabajo sin alterar equilibrios macroeconómicos. En segundo término, los empleadores ya no pueden reclutar mujeres y jóvenes, como hacían en el pasado. Ese yacimiento de nuevos trabajadores se ha truncado debido a los cambios demográficos y sociales recientes.

Desde el punto de vista del capital social y de la red social, el grupo étnico y las redes sociales del inmigrante son claves para entender sus patrones de movilidad ocupacional y de integración laboral. Sin embargo, el tipo de impacto varía de acuerdo a los diferentes enfoques teóricos: mientras que algunos investigadores argumentan que la cohesión del grupo étnico y extensas redes sociales facilitan la búsqueda de trabajo y la movilidad, ${ }^{21}$ otros consideran que los empleos encontrados a través de estos mecanismos tienden a ser de menor calidad y

20. PIORE, Michael, Birds of Passage..., Op. Cit.; SASSEN, Saskia, "La movilidad del trabajo y del capital. Un estudio de la corriente internacional de la inversión $y$ del trabajo", MTAS, Madrid, 1993. MOULIERBOUTANG, Yann, "L'avenir des migrations vers 1'Europe: changer de système migratoire et de paradigm", en BRIBOSIA, Emmanuelle y REA, Andrea (directoras) Les nouvelles migrations. Un enjeu européen, Complex, Bruselas, 2002, pp. 75-91.

21. MULLAN, Brendan P., "The impact of social networks on the occupational status of migrants", en International Migration, Grand-Saconnex-Suiza, 1989, V. 27, No 1, pp. 69-86; YAMAUCHI, Futoshi y TANABE, Sakiko, "Nonmarket networks among migrants: Evidence from metropolitan Bangkok", en Journal of Population Economics, Thailand, 2008, V. 21, No 3, pp. 649-664. 
limitados a nichos específicos de entrada en el mercado de trabajo, reduciendo así los salarios y las posibilidades de movilidad ulterior. ${ }^{22}$ Por otra parte, los estudios confirman que el acceso a las redes sociales de nativos aumenta la probabilidad de empleo de los inmigrantes, por ejemplo, a través del matrimonio con una persona autóctona. ${ }^{23}$

Por último, investigaciones recientes realizadas desde la perspectiva teórica de Pierre Bourdieu han confirmado que el capital social de los inmigrantes es crucial para su integración en el mercado de trabajo, más allá de su estatus legal y nivel educativo. No obstante, ello depende de que el capital social sea adecuado, ya que cada segmento del mercado de trabajo es un campo con reglas de admisión y movilidad específicas. $^{24}$

\section{Los motivos para la emigración}

El proyecto migratorio define los motivos y objetivos de la migración, que también afec-

22. PATEL, Krishna y VELLA, Francis, "Immigrant networks and their implications for occupational choice and wages", en Review of Economics and Statistics, Cambridge, 2013, V. 95, № 4, pp. 1249-1277; PORTES, Alejandro, "Social Capital: Its Origins and Applications in Modern sociology", en Annual Review of Sociology, Palo Alto, 1998, 24, pp. 1-24. Para el caso de España ver: VONO, Daniela y VIDAL, Elena, "The impact of informal networks on labour mobility: Immigrants' first job in Spain”, en Migration Letters, London-Reino Unido, 2012, V. 9, № 3, pp. 237-247.

\section{FURTADO, Delia y THEODOROPOULOS,}

Nikolaos, "Why does intermarriage increase immigrant employment? The role of networks", en The B.E. Journal of Economic Analysis \& Policy, 2010, V.10, No 1, pp. 1-33.

24. AKKAYMAK, Guliz, "A Bourdieuian Analysis of Job Search Experiences of Immigrants in Canada", en Migration E Integration, 2017, № 18, pp. 57-674. $\tan$ a las trayectorias laborales en la sociedad de acogida. ${ }^{25}$ Por un lado, los refugiados y los migrantes económicos que se desplazaron con sus familias de descendencia suelen tener un mayor declive en su posición ocupacional, desde el último trabajo de origen hasta el primer trabajo al llegar. Sin embargo, más adelante, hay una mayor mejora de la ocupación y el ingreso: el modelo en forma de " $U$ " es más profundo. Por otra parte, los migrantes económicos que se desplazan solos sufren menos movilidad descendente en el momento de su llegada, pero su posterior movilidad ascendente es más lenta.

Las diferencias de ingresos (directos e indirectos) entre los países de origen y destino operan habitualmente como "efecto llamada". Así, es probable la ocupación inicial de los inmigrantes sea el resultado de decisiones guiadas por el objetivo de obtener ganancias económicas inmediatas para satisfacer necesidades familiares o para financiar el viaje, incluso a costa de un deterioro de las condiciones de trabajo. ${ }^{26}$

La literatura también se refiere las diferencias significativas en la movilidad laboral según las

25. SANTACREU, Oscar, BALDONI, Emiliana y ALBERT, Ma Carmen, "Deciding to move: migration projects in an integrating Europe", en RECCHI, Ettore y FAVELL, Adrian (editores) Pioneers of European integration: Citizenship and mobility in the EU, Edward Elgar Publishing Limited, Cheltenham, 2009, pp. 52-71.

26. KOSSOUDJI, Sherrie y COBB-CLARK, Deborah, "IRCA's impact on the occupational concentration and mobility of newly-legalized Mexican men", en Journal of Population Economics, Thailand, 2000, V. 13, No 1, pp. 81-98. KOGAN, Irena, "Last hired, first fired? The unemployment dynamics of male immigrants in Germany", en European Sociological Review, Mannheim, 2004, V. 20, No 5, pp. 445-461; REDSTONE AKRESH, Iliana, "Occupational mobility among legal immigrants to the United States", en International Migration Review, Staten Island, 2006, V. 40, № 4, pp. 854-884. 
características de los hogares, el asentamiento temporal o permanente de los migrantes $y$, principalmente, las desigualdades de género en el mercado de trabajo. En este sentido, algunos autores han demostrado que la movilidad ascendente es más alta para los hombres que para las mujeres. ${ }^{27} \mathrm{La}$ Teoría de Interseccionalidad argumenta que las mujeres se enfrentan a una superposición de desventajas en la cual el grupo étnico y la case social de pertenencia se articulan con el género. ${ }^{28}$

"Las decisiones de la migración familiar", 29 "el modelo de inversión familiar" 30 o "la racionalidad familiar" 31 también podrían explicar esta variación en los patrones de movilidad entre hombres y mujeres inmigrantes. Después de establecerse en el país receptor, los ingresos de las mujeres inmigrantes casadas tienden a dis-

27. ANGUIANO TÉLLEZ, Ma. Eugenia, "Emigración reciente de latinoamericanos a España: trayectorias laborales y movilidad ocupacional", en Papeles de población, Toluca-México, 2002, V. 33, No 8, pp. 101-115.

28. RAIJMAN, Rebeca, SEMYONOV, Moshe, "Gender, ethnicity, and immigration: Double disadvantage and triple disadvantage among recent immigrant women in the Israeli labor market", en Gender E' Society, 1997, V. 11, No 1, pp. 108-125; FLIPPEN, Chenoa A., "Intersectionality at work: Determinants of labor supply among immigrant Hispanic women", en Population Association of American 2013 annual meeting program, 2013. Disponible en: <http://paa2013.princeton.edu/ abstracts/131053>.

29. MINCER, Jacob, "Family migration decisions", en Journal of Political Economy, Chicago, 1978, V. 86, $\mathrm{N}^{\circ} 5$, pp. 749-773.

30. LONG, James E. "The effect of Americanization on earnings: Some evidence for women”. Journal of Political Economy, Chicago, 1980, V. 88, No 3, pp. 620-629.

31. BAKER, Michael y BENJAMIN, Dwayne, "The role of the family in immigrants labor-market activity: An evaluation of alternative explanations", en American Economic Review, Nashville, 1997, V. 87, No 4, pp. 705727. minuir con el tiempo, incluso es probable que abandonen temporalmente el mercado laboral. En términos generales, esto se debe a que suelen reducir el tiempo dedicado al empleo e incrementar el tiempo dedicado a las actividades reproductivas debido a la dificultad de conciliar la vida laboral y familiar. ${ }^{32}$

\section{Las migraciones de argentinos a España y sus contextos de recepción}

La literatura ha identificado tres períodos importantes en "la formación de la España inmigrante". ${ }^{33}$ El primero hasta 1985, el segundo desde 1986 hasta 1999 y el tercero desde 2000 hasta 2008. Estas etapas coinciden con los flujos migratorios de argentinos más significativos: los exiliados (1975-1983), los primeros migrantes económicos (hasta 1999) y la migración masiva (2000-2007). ${ }^{34}$ A partir de 2008, comenzó un nuevo ciclo migratorio en España en el marco de la crisis económica, caracterizado por la emigración de nativos y el retorno y la re-emigración de inmigrantes extranjeros. Una pauta que también siguieron los inmigrantes argentinos, pero excede los objetivos de este trabajo. ${ }^{35}$

32. DULEEP, Harriet O. y SANDERS, Seth, "The decision to work by married immigrant women", en Industrial and Labor Relations Review, 1993, V. 46, No 4, pp. 677-690. DULEEP, Harriet y DOWHAN, Daniel J., "Revisiting the family investment model with longitudinal data the earnings growth of immigrant and U.S.-born women", en IZA Discussion Paper, Bonn, 2002, № 568 .

33. CACHÓN, Lorenzo, La España inmigrante: marco discriminatorio, mercado de trabajo y politicas de integración, Anthropos, Barcelona, 2009.

34. ACTIS, Walter; ESTEBAN, Fernando Osvaldo, "Argentinos en España...", Op. Cit.

35. ESTEBAN, Fernando Osvaldo, Cuatro décadas..., 
De acuerdo con la literatura española, ${ }^{36}$ estas etapas constituyen "contextos de recepción" diferentes debido a las transformaciones ocurridas en aspectos determinantes para la inserción de los inmigrantes. A continuación describiremos en trazos gruesos cada uno de ellos.

En la primera etapa (hasta 1985), la inmigración extranjera era fundamentalmente europea (65\% de los residentes en 1981) y, en menor medida latinoamericana (18\%). Los europeos eran en su mayoría "turistas residenciales" retirados de la actividad económica o trabajadores cualificados en empresas de sus países de origen. Los latinoamericanos, en cambio, habían arribado huyendo de dictaduras militares. Este último era el caso de gran parte del stock de argentinos. Considerando residentes y nacionalizados españoles, se puede estimar que a mediados de los ochenta residían en España en torno a 40.000 personas de origen argentino (véase, Tabla 1), la mayoría arribada durante la dictadura militar y una pequeña parte anteriormente, en un "goteo" eran descendientes de españoles emigrados a la Argentina. ${ }^{37}$

Como sostienen los estudios sobre el exilio argentino en España, ${ }^{38}$ este grupo era un conglomerado heterogéneo, aunque la mayoría podía

Op. Cit.; CERRUTI, Marcela y MAGUID, Alicia, "Crisis económica en España y el retorno de inmigrantes sudamenricanos”, en Migraciones Internacionales, 2016, V., № 3, pp. 155-189.

36. CACHÓN, Lorenzo, "Marco institucional de la discriminación y tipos de inmigrantes en el mercado de trabajo en España”, en Revista Española de Investigaciones Sociológicas, Madrid, 1995, № 69, pp. 105-124; CACHÓN, Lorenzo. La España inmigrante..., Op. Cit.

37. ACTIS, Walter; ESTEBAN, Fernando Osvaldo, "Argentinos en España...", Op. Cit.

38. Mira Delli-Zotti, “¿Sobrevivir o vivir...”, Op. Cit.; JENSEN, Silvina, La provincia flotante..., Op. Cit. adscribirse a los tramos superiores de la clase media en Argentina. Vale remarcar que, precisamente, este grupo social estaba compuesto por la primera y la segunda generación de inmigrantes internos y extranjeros, limítrofes y europeos, provenientes de sectores sociales de diverso origen (asalariados, cuentapropistas y profesionales) que no tenían la intención de emigrar del país para satisfacer sus expectativas de movilidad social. ${ }^{39} \mathrm{Al}$ contrario, la clase media, que en 1970 constituía el 44,9\% de la población argentina, se fue constituyendo mediante un proceso de movilidad social ascendente en el marco de los modelos de acumulación justicialista y desarrollista entre las décadas de 1940 y $1970 .{ }^{40}$ En este proceso, fueron determinantes el acceso a la formación (formal e informal) y un marco normativo en el cual el progreso personal coronaba el esfuerzo.

Volviendo a España, el período comprendido desde la muerte de Franco hasta mediados de los '80 fue un contexto de recepción favorable desde el punto de vista económico y político para los argentinos y, en general, para los inmigrantes latinoamericanos. ${ }^{41}$ En primer lugar, se consolidaron importantes transformaciones en el mercado de trabajo. Una gran movilidad ocupacional hacia los servicios avanzados, el aumento de categorías ocupacionales de cuadros superiores, la incorporación laboral de la mujer y la creación de empleo público por la nueva organización territorial del Estado y la creación del Estado de Bienestar, ${ }^{42}$ favore-

39. TORRADO, Susana, Estructura social de la Argentina (1945-1983), Ediciones de la Flor, Buenos Aires, 1994.

40. Ibídem

41. HERRANZ, Yolanda, "La inmigración latinoamericana en distintos contextos de recepción", en Migraciones, Madrid, 1998, 3, pp. 31-51.

42. GARRIDO, Luis y GONZÁLEZ, Juan, “Mercado 
cieron la incorporación inicial (o la movilidad posterior) de los argentinos en categorías de técnicos y profesionales. ${ }^{43}$ No obstante, el capital cultural incorporado e institucionalizado que portaban los exiliados fue un factor fundamental para acceder a estas nuevas posiciones.

En segundo lugar, y desde un punto de vista político, el contexto de recepción también fue favorable para los exiliados en dos aspectos principales: a) porque durante "la época de la transición" se emulaban sentimientos de solidaridad por la identificación del exilio latinoamericano con el español, como en relación a la clase trabajadora en su conjunto y, en general, con el auge de ideologías políticas de izquierda; b) porque según la política migratoria vigente hasta 1985 , basada en vínculos coloniales, los inmigrantes originarios de Iberoamérica no necesitaban un permiso previo de trabajo y residencia en España. Una vez en el país, bastaba la inscripción en el Ministerio de Trabajo para obtener un permiso de residencia. ${ }^{44}$

De acuerdo con Cachón, ${ }^{45}$ la "segunda etapa de la España inmigrante" comenzó con la entrada en vigor de la primera Ley de Extranjería (LO 7/1985) y su Reglamento. ${ }^{46}$ En ese

de trabajo, ocupación y clases sociales", en GONZÁLEZ, Juan y REQUENA y DÍAZ DE REVENGA, Manuel (editores) Tres décadas de cambio social en España, Alianza, Madrid, 2005, pp. 81-126.

43. ACTIS, Walter; ESTEBAN, Fernando Osvaldo, "Argentinos en España...”, Op. Cit.

44. Ley 118/1969, de 30 de diciembre, y Orden Ministerial del 15 de enero de 1970.

45. CACHÓN, Lorenzo. La España inmigrante...,Op. Cit.

46. Real Decreto 1119/1986, de 26 de mayo, por el que se aprueba el reglamento de ejecución de la Ley Orgánica (en adelante, L.O.) 7/1985, de $1^{\circ}$ de julio, sobre derechos y libertades de los extranjeros en España. (BOE, 12 de momento se puso en marcha un rígido control de entradas que debía ser siempre previa obtención de visado en el país de origen y a una oferta de trabajo para España. ${ }^{47}$ La normativa dio comienzo a un férreo mecanismo de discriminación institucional que asignó derechos diferentes a extranjeros comunitarios (entonces Comunidad Económica Europea) y extranjeros no comunitarios. Según Herranz, ${ }^{48}$ los inmigrantes latinoamericanos fueron los más perjudicados por el nuevo marco regulador.

En este período, el volumen y la composición de los flujos permiten hablar de una "nueva inmigración". "Nueva" por sus zonas de origen y el nivel de desarrollo de esas zonas (principalmente Marruecos, China, Filipinas, Perú, Cuba y R. Dominicana,), por sus culturas y sus religiones no cristianas, por sus rasgos fenotípicos que la hacían fácilmente perceptible entre la población, por las motivaciones económicas de la migración y por la existencia de un "efecto llamada" desde el mercado de trabajo español. ${ }^{49}$

Los flujos de argentinos se caracterizaron por el retorno de los exiliados políticos y por una moderada inmigración laboral que fluctuó de acuerdo a la coyuntura socioeconómica en $\mathrm{Ar}$ gentina. Se registraron dos picos de salidas: durante el proceso hiperinflacionario (1989-

junio).

47. AJA, Eliseo, "La evolución sobre normativa sobre inmigración", en AJA, Eliseo y ARANGO, Joaquín (editores) Veinte años de inmigración en España. Perspectivas jurídica y sociológica (1985-2005), Fundació CIDOB, Barcelona, 2006, pp. 17-44.

48. HERRANZ, Yolanda. "La inmigración latinoamericana...”, Op. Cit.

49. CACHÓN, Lorenzo, La España inmigrante..., Op. Cit, p. 110. 
1991) y al comienzo de la crisis del modelo de convertibilidad (1998-1999). Recordemos que hasta la década de 1980, la relación entre crecimiento económico y absorción productiva de la fuerza de trabajo, junto con un Estado de bienestar incipiente, aunque limitado e imperfecto, fueron los mecanismos que alimentaron las expectativas de movilidad social de importantes sectores de la población argentina. Pero el modelo de acumulación aperturista instaurado por la dictadura militar, ${ }^{50} \mathrm{y}$ su consolidación durante los años '90, implicaron nuevas formas de relación de los hogares con el mercado de trabajo y con el Estado, que trastocaron fuertemente la estructura social argentina ${ }^{51}$ La crisis de la sociedad salarial, ${ }^{52} \mathrm{el}$ profundo proceso de reconversión productiva (privatización de empresas públicas, desregulación del comercio exterior, descentralización y reducción de la administración pública, especialización primario - exportadora), junto con el progresivo desmantelamiento y mercantilización de los servicios sociales, se tradujo en un aumento de la pobreza y la vulnerabilidad social y, asociado a ésta, una fragmentación de las clases medias que buscaron en la emigración una forma de mantener la posición. ${ }^{53}$

En el fin de siglo anterior, se estima que la

50. TORRADO, Susana, Estructura social..., Op. Cit.

51. Sobre Estado, cuestión social y política sociales en la década de 1990 ver GRASSI, Estela, Politicas y problemas sociales en la sociedad neoliberal: la otra década infame (I), Espacio, Buenos Aires, 2003.

52. ALONSO, Luis Enrique, Trabajo y ciudadania: estudios sobre la crisis de la sociedad salarial, Trotta, Madrid, 1999.

53. Sobre los rasgos generales de este proceso ver SVAMPA, Maristella, "Las clases medias en Argentina", en SVAMPA, Maristella, Los que ganaron. La vida en los countries y barrios privados. Biblos, Buenos Aires, 2001, pp. 19-48. colonia argentina en España sumaba cerca de 70.000 personas (véase, Tabla 1). ${ }^{54}$ Los "recién llegados" se encontraron con un mercado de trabajo en expansión que operaba como un formidable "factor de atracción". La demanda de mano de obra tenía origen, por un lado, en la expansión de los servicios, en particular los servicios públicos, a consecuencia de la reforma y descentralización de la administración pública y la expansión del Estado de Bienestar. También se extendía a las grandes empresas, algunas recién privatizadas (telecomunicaciones, banca, energía, metalmecánicas). Era una demanda de trabajadores con niveles de cualificación medio y alto para ocupar empleos de calidad. ${ }^{55}$ Por otro lado, y una vez superada la crisis económica de mediados de los '90, despuntó una demanda progresiva de trabajadores menos cualificados para ocupar empleos en el segmento secundario del mercado de trabajo (sobre todo construcción de viviendas e infraestructuras y servicios de proximidad). Se consolidaba, así, un cambio de paradigma productivo iniciado décadas atrás. ${ }^{56}$ Es importante resaltar que este fue período en el cual la mujer española se incorporó masivamente al mercado de trabajo ${ }^{57}$, externalizando tareas de

54. ACTIS, Walter; ESTEBAN, Fernando Osvaldo, "Argentinos en España...", Op. Cit.

55. Entre 1985 y 1991 se crearon más de dos millones de empleos no agrarios, de los cuales casi la mitad eran empleos de nueva clase media, es decir, empleos calificados en la industria y los servicios. GARRIDO, Luis y GONZÁLEZ, Juan, “Mercado de trabajo...”, Op. Cit., p. 91.

56. Un indicador de referencia de esta transformación es que entre 1980 y 2000 la población activa en la agricultura se redujo del $17,1 \%$ al 7,0\% y en la industria del 26,0\% al 18\%, mientras se incrementó en los servicios del 42\% al 58,7\% (Instituto Nacional de Estadística de España INE, Encuesta de Población Activa (en adelante, EPA), segundos trimestres).

57. La tasa de actividad de las mujeres pasó de 28,5\% en 
cuidado en los hogares que fueron desempeñadas principalmente por mujeres inmigrantes. ${ }^{58}$

La tercera etapa de la "España inmigrante" comenzó en 2000 y continuó hasta 2008. Fue el período de mayor incremento de la inmigración extranjera en España. En tan solo ocho años el número de inmigrantes se multiplicó por seis (de 923.879 a 5.268.762); representaban el 2,3\% de una población de 40.5 millones de habitantes, mientras en 2008, sobre un total de 46,1 millones, suponían el 11,4\% (véase, Tabla 1). También se modificó la composición de la inmigración. Entre 2000 y 2004 las llegadas masivas procedían principalmente de $\mathrm{La}^{-}$ tinoamérica (Ecuador, Colombia, Bolivia, Argentina) y luego de Europa del Este (Rumanía y Bulgaria). En esta época la inmigración se transformó en un problema social y comenzó a ocupar un lugar destacado en las agendas políticas, mediáticas y académicas.

Esta etapa coincidió con las cifras más altas de inmigración de argentinos: de 70.500 empadronados en 1999 se pasó a 295.000 en 2008 (véase, Tabla 1). La llegada masiva de una inmigración "improvisada" que buscaba un refugio a la crisis, junto con la política de "cierre migratorio" operada por las autoridades españolas, produjeron un importante crecimiento de inmigrantes en situación irregular (que llegaron a ser el $73 \%$ de los empadronados en 2002).

1985 a 39,6\% en 1999 ( $2^{\circ}$ cuatrimestre, EPA).

58. Sobre los patrones de inserción laboral de las mujeres autóctonas ver, entre otros, CEBRIÁN LÓPEZ, Inmaculada, MORENO RAYMUNDO, Gloria, "La situación de las mujeres en el mercado de trabajo español. Desajustes y retos", en Economía industrial, Madrid, 2008, No 367, p. 121-137.
Esta emigración masiva tuvo origen en la catástrofe social que experimentó el país a consecuencia de la crisis del modelo de acumulación neoliberal que estalló en diciembre de 2001. ${ }^{59}$ Se registraron niveles de pobreza superiores al $50 \%$, una proporción similar de población activa tenía problemas de empleo, se produjo una fuerte devaluación de la moneda, una restricción del circulante, el "corralito" sobre los depósitos bancarios... La crisis económica y social redundó en una crisis política e institucional de enormes dimensiones. ${ }^{60}$

La decisión de emigrar a España como estrategia para sortear la pobreza o la movilidad social descendente también se explica por la influencia de un nuevo marco de valores en la sociedad argentina. Las soluciones a los problemas sociales dejaron de pasar por las instituciones formales o las instancias colectivas (partidos políticos, sindicatos, asociaciones, etc.) para asumirse individualmente. Este proceso se inscribió en un contexto de efectivo deterioro de las instituciones propio del desmantelamiento del modelo de regulación asociado al régimen fordista y, en relación a éste, a una reformulación del rol del individuo en la sociedad. Como consecuencia de ello, en el nuevo orden social, "el bienestar ya no aparecía como un derecho, sino como una oportunidad". ${ }^{61}$

59. Para una visión holística de la "salida neoliberal" (1989-2001) ver: SVAMPA, Maristella, La sociedad excluyente. La Argentina bajo el signo del neoliberalismo, Taurus, Buenos Aires, 2005.

60. Para una descripción de las consecuencias sociales de la crisis ver, entre otros, ROMERO, Luis A., La crisis argentina: una mirada al siglo XX, S.XXI, Buenos Aires, 2003.

61. ALONSO, Luis E., "Ciudadanía, sociedad del trabajo y Estado de Bienestar: los derechos sociales en la era de la fragmentación”, en PÉREZ LEDESMA, Manuel (compilador) Ciudadanía y democracia, Pablo Iglesias, Madrid, 2000, p. 176. 
Tabla 1. España. Población total, extranjeros y argentinos (1986-2008)*

\begin{tabular}{rrrrrr}
\hline \multirow{2}{*}{ Año } & \multirow{2}{*}{ Población total } & \multicolumn{2}{c}{ Extranjeros } & \multicolumn{2}{c}{ Argentinos } \\
\cline { 3 - 6 } & & N & (\%) & \multicolumn{1}{c}{ N } & (\%) \\
\hline 1986 & 38.437 .362 & 241.971 & 0,7 & 41.500 & 17,2 \\
1999 & 40.202 .160 & 748.954 & 1,86 & 70.491 & 9,4 \\
\hline 2000 & 40.499 .791 & 923.879 & 2,28 & 84.872 & 9,2 \\
\hline 2001 & 41.116 .842 & 1.370 .657 & 3,33 & 118.903 & 8,7 \\
\hline 2002 & 41.837 .894 & 1.977 .946 & 4,73 & 191.653 & 9,7 \\
\hline 2003 & 42.717 .064 & 2.664 .168 & 6,24 & 226.548 & 8,5 \\
\hline 2004 & 43.197 .684 & 3.034 .326 & 7,02 & 260.386 & 8,6 \\
\hline 2005 & 44.108 .530 & 3.730 .610 & 8,46 & 271.444 & 7,3 \\
\hline 2006 & 44.708 .964 & 4.144 .166 & 9,27 & 272.985 & 6,6 \\
\hline 2007 & 45.200 .737 & 4.519 .554 & 10,0 & 290.281 & 6,4 \\
\hline 2008 & 46.157 .822 & 5.268 .762 & 11,4 & 295.401 & 5,6 \\
\hline
\end{tabular}

*En 1986 y 1999 el stock de argentinos está compuesto por residentes y nacionalizados, a partir de 2000 por personas nacidas en Argentinas inscriptas en padrones municipales

Fuente: Padrón de Habitantes, Registro de Residentes, Instituto Nacional de Estadística de España (INE).

Los argentinos que arribaron a partir de 2000 hallaron un país con un crecimiento económico sostenido de extraordinarias dimensiones, ${ }^{62}$ con un aumento poblacional significativo $(5,6$ millones de personas, de las cuales 4,9 eran nuevos trabajadores reales o potenciales) y una gran reducción del desempleo (del 20\% en 1996 hasta el 9\% en 2007). Asociado a esto,

62. Desde 1996 la economía española venía experimentado un crecimiento medio anual del 3,5\%, muy por encima del crecimiento medio del PIB de los socios de la zona euro $(2,1 \%)$, de la UE-25 $(2,3 \%)$ e incluso superior al registrado en EEUU (3,3\%). OFICINA ECONÓMICA DEL PRESIDENTE, Inmigración y economía española: 1996-2006, Presidencia del Gobierno, Madrid, 2006, p. 1. un mercado de trabajo en ebullición ( 5 millones de nuevos empleos), sobre la base de un modelo de desarrollo de baja productividad e intensivo en mano de obra, que protagonizó el boom económico del nuevo siglo con la construcción, el turismo y los servicios a las empresas como sectores estrella. Con este modelo se fue conformando una estructura productiva etnofragmentada. ${ }^{63}$

Durante este período los fundamentos de la política migratoria (L.O. 4/2000; L.O. 8/2000; L.O. 14/2003) siguieron siendo igual-

63. CACHÓN, Lorenzo. La España inmigrante..., Op. Cit. 
mente restrictivos. No obstante, se pusieron en marcha medidas que favorecieron la integración de los extranjeros como los procesos extraordinarios de regularización, la agilización de la homologación de credenciales educativas, una mayor flexibilidad para la obtención de la nacionalidad española (sobre todo para Latinoamericanos) y el acceso al sistema sanitario y educativo en las mismas condiciones que los nativos. ${ }^{64}$

Antes de finalizar el apartado sería de interés mencionar algunas características comunes a los tres flujos migratorios. ${ }^{65}$ Desde la década de 1970 hasta la actualidad, las migraciones de argentinos a España siempre presentaron un volumen similar de hombres y mujeres. A consecuencia de ello, la existencia de familias transnacionales fue escasa entre los argentinos. ${ }^{66} \mathrm{La}$ estructura etaria refleja la huella demográfica de la inmigración en la que ha predominado un elevado componente de población activa. Cada nuevo flujo migratorio aportó efectivos jóvenes, sin embargo, se trata de uno de los colectivos nacionales más envejecidos debido a la "antigüedad de la migración" y a que los migrantes se desplazaron a edades más avanzadas. Respecto al nivel educativo, los inmigrantes argentinos en España presentan un mejor perfil que el conjunto de la población en Argentina y en España. Ello significa que, más allá de algunas diferencias mínimas

64. Para profundizar sobre estos temas ver, entre otros: AJA, Eliseo y ARANGO, Joaquín (editores) Veinte años..., Op. Cit.

65. ESTEBAN, Fernando Osvaldo, Cuatro décadas..., Op. Cit.

66. CERRUTI, Marcela y MAGUID, Alicia, "Familias divididas y cadenas globales de cuidados: la migración sudamericana a España”, en CEPAL. Serie Políticas Sociales, 2010, № 163. entre flujos ${ }^{67}$ existe una proporción menor de inmigrantes con educación primaria o menos y una proporción mayor con educación superior, especialmente en el caso de las mujeres.

A partir de lo visto hasta aquí, el artículo se propone contrastar las siguientes hipótesis: a) siguiendo a Chiswick, ${ }^{68}$ la inserción laboral inicial de los inmigrantes en España se produjo en categorías inferiores a la que tenían en su última ocupación en Argentina; b) los flujos migratorios más antiguos, particularmente el del exilio, ingresaron al mercado de trabajo español en mejores condiciones que los más recientes. ${ }^{69}$

\section{Fuentes y metodología de la investigación}

Un problema en discusión en la literatura sobre inserción y movilidad laboral son los indicadores utilizados para medirla. En la sociedad industrial moderna, generalmente, el desempeño de una profesión de "cuello azul" o de "cuello blanco" es uno de los indicadores más contrastados. ${ }^{70}$ Sin embargo, hoy día no es

67. Si bien es cierto que los exiliados presentan un mejor perfil educativo, éste se alcanzó en España después de la migración. ESTEBAN, Fernando Osvaldo, ACTIS, Walter, "Migración de personas cualificadas de Argentina a España ¿¿Una nueva fuga de cerebros?”, en LUCHILO, Lucas (coordinador) Más allá de la fuga de cerebros. Movilidad, migración y diásporas de argentinos calificados, Eudeba, Buenos Aires, 2011, pp. 117-168..

68. CHISWICK, Barry, "The effect of Americanization...”, Op. Cit.

69. HERRANZ, Yolanda, "La inmigración latinoamericana...”, Op. Cit.

70. GOLDTHORPE, John H., LLEWELLYN, C., PAYNE, G., Class structure and social mobility in modern Britain, Oxford University Press, Oxford, 1980. 
suficiente ya que, como ha demostrado Braverman, ${ }^{71}$ muchos trabajos de cuello blanco se han descualificado a través de la automatización y la taylorización del proceso productivo. Por tanto, renunciamos a considerar un solo indicador sintético de inserción y movilidad laboral y optamos por realizar una triangulación de tres indicadores provistos por la ENI: la situación profesional, la situación sociolaboral y la duración del contrato. ${ }^{72}$

La "situación profesional" clasifica a los trabajadores según la propiedad de los medios de producción y, por extensión, según la autoridad y control del proceso productivo explorando si el trabajador ha ganado o perdido control y autoridad sobre su trabajo y el de otros. El indicador define las categorías de asalariado, autónomo y empresario. Habitualmente suele denominarse "relación de dependencia" en la bibliografía y algunas fuentes de datos.

La "situación socio-laboral" corresponde a una subdivisión de la población ocupada en cierto número de agregados distintos en estatus o prestigio ocupacional y recompensas materiales. La elección de este tipo de escala, a diferencia de otra basada en categorías ocupacionales más usual en sociología, se basa en que no importan tanto el número de posiciones sucesivas que ocupan los individuos, sino cómo los cambios entre segmentos del mercado de trabajo implican posicionamientos objetivables y tipificables en las condiciones de empleo $\mathrm{y}$ en las perspectivas subjetivas de los actores. La escala contempla cinco posiciones: manual

71. BRAVERMAN, Harry, Trabajo y capital monopolista: la degradación del trabajo o en el siglo XX, Siglo XXI, Madrid, 1975.

72. Los nombres de los indicadores se reproducen tal y como han sido enunciados en la ENI. cualificado, manual no cualificado, administrativo, técnico y directivo.

El tercer indicador mide la duración del contrato y se refiere así a una mayor o menor estabilidad laboral. Un contrato indefinido tiene un componente "prospectivo", como es la posibilidad de un aumento salarial, un ascenso o acceso a la formación continua, que no existe en un contrato temporal.

Pero antes de adentrarnos en el análisis de los datos, es necesario describir con mayor precisión la fuente. En lo que respecta a este trabajo, la explotación de los microdatos de la ENI permitió, por un lado, discriminar a la población nacida en Argentina sin perder representación estadística, y por otro, conocer su trayectoria laboral desde el último empleo en Argentina al primero en España, incluyendo aquellos que se desarrollaron en la economía sumergida.

Los datos fueron discriminados a partir de la variable independiente "período de llegada” porque se comprobó en una investigación anterior que esta variable era relevante para entender la inserción socioeconómica de los inmigrantes argentinos en España. ${ }^{73}$ De este modo distinguimos tres flujos migratorios cuantitativamente significativos y con características propias bien definidas: "el flujo del exilio" entre 1976 y 1983, los emigrados durante el período de inestabilidad económica y reformas estructurales (entre 1984 y 1999) y el "flujo del corralito" durante la etapa de crisis y estabilización entre 2000 y 2007. Ello ha permitido realizar un análisis comparativo entre los diferentes flujos acerca de la inserción en el mercado de trabajo español y de la movilidad laboral trasnacional.

73. ACTIS, Walter; ESTEBAN, Fernando Osvaldo, "Argentinos en España...", Op. Cit. 
Tabla 2. España. Población nacida en Argentina, de 16 y más años, según período de llegada y edad al llegar. A $1^{\circ}$ de enero de 2007.

\begin{tabular}{|c|c|c|c|c|c|c|c|c|}
\hline \multirow{3}{*}{ Añollegada } & \multicolumn{4}{|c|}{$\mathbf{N}$} & \multicolumn{4}{|c|}{$(\%)$} \\
\hline & \multicolumn{3}{|c|}{ Edad } & \multirow{2}{*}{ Total } & \multicolumn{3}{|c|}{ Edad } & \multirow{2}{*}{ Total } \\
\hline & $0-15$ & $16-64$ & $65-69$ & & $0-15$ & $16-64$ & $65-69$ & \\
\hline * Hasta 1975 & 11.492 & 5.617 & 0 & 17.109 & 67 & 33 & 0 & 100 \\
\hline 1976-83 & 3.466 & 8.022 & 0 & 11.488 & 30 & 70 & 0 & 100 \\
\hline 1984-99 & 11.333 & 37.427 & 1.311 & 50.071 & 23 & 75 & 3 & 100 \\
\hline $2000-07$ & 7.322 & 141.003 & 3.100 & 151.425 & 5 & 93 & 2 & 100 \\
\hline Total & 33.613 & 192.069 & 4.411 & 230.093 & 15 & 83 & 2 & 100 \\
\hline Sin información & & & & 1.527 & & & & \\
\hline Población total & & & & 231.620 & & & & \\
\hline
\end{tabular}

Fuente: Instituto Nacional de Estadística de España (INE), Encuesta Nacional de Inmigrantes (ENI) y elaboración propia.

La muestra estuvo compuesta por 793 personas nacidas en Argentina que tenían en ese momento 16 años o más. El diseño de la ENI excluyó a los menores de 15 años bajo el supuesto de que emigraron acompañando a sus padres. Nuestra exploración, en cambio, contempló un universo distinto. Consideramos sociológicamente más interesante circunscribir el análisis a la población que emigró de Argentina a España en edades comprendidas entre los 16 y 64 años, es decir, personas económicamente activas y con autonomía para tomar la decisión de emigrar. De ese modo, no sólo quedaron excluidas 33.613 personas entre $0 \mathrm{y}$ 15 años, sino también 4.411 con 65 o más años y 1.527 casos que no ofrecían información sobre la edad al emigrar. Con todo, el universo representado en nuestra explotación ascendió a 192.069 personas, el $83 \%$ de la población total compuesta por 231.620 personas (véase, Tabla 2).

\section{La situación en el último empleo en Argentina}

Durante la época del exilio emigraron a España mayoritariamente personas que se encontraban trabajando (75\%), aunque también fue singular la proporción de estudiantes (16\%) (véase, Gráfico 1). En esta oleada, las personas que sólo realizaban labores domésticas eran, en comparación, escasas. Esta distribución de actividades se asemeja a la de una migración económica (por su componente de activos), pero también tiene características específicas del exilio como una participación elevada de estudiantes y escasa de personas dedicadas a labores domésticas, teniendo en cuenta la división sexual del trabajo en los hogares en una época en la que primaba un modelo reproductivo del tipo wife housekeeper y male breadwinner.

Los flujos económicos que arribaron a partir de 1984 también tuvieron una elevada propor- 
Gráfico 1. España. Población nacida en Argentina, emigrada entre los 16 y 64 años, según tipo de actividad desempeñada en Argentina antes de emigrar y período de llegada. Al 1 de enero de 2007. En porcentajes

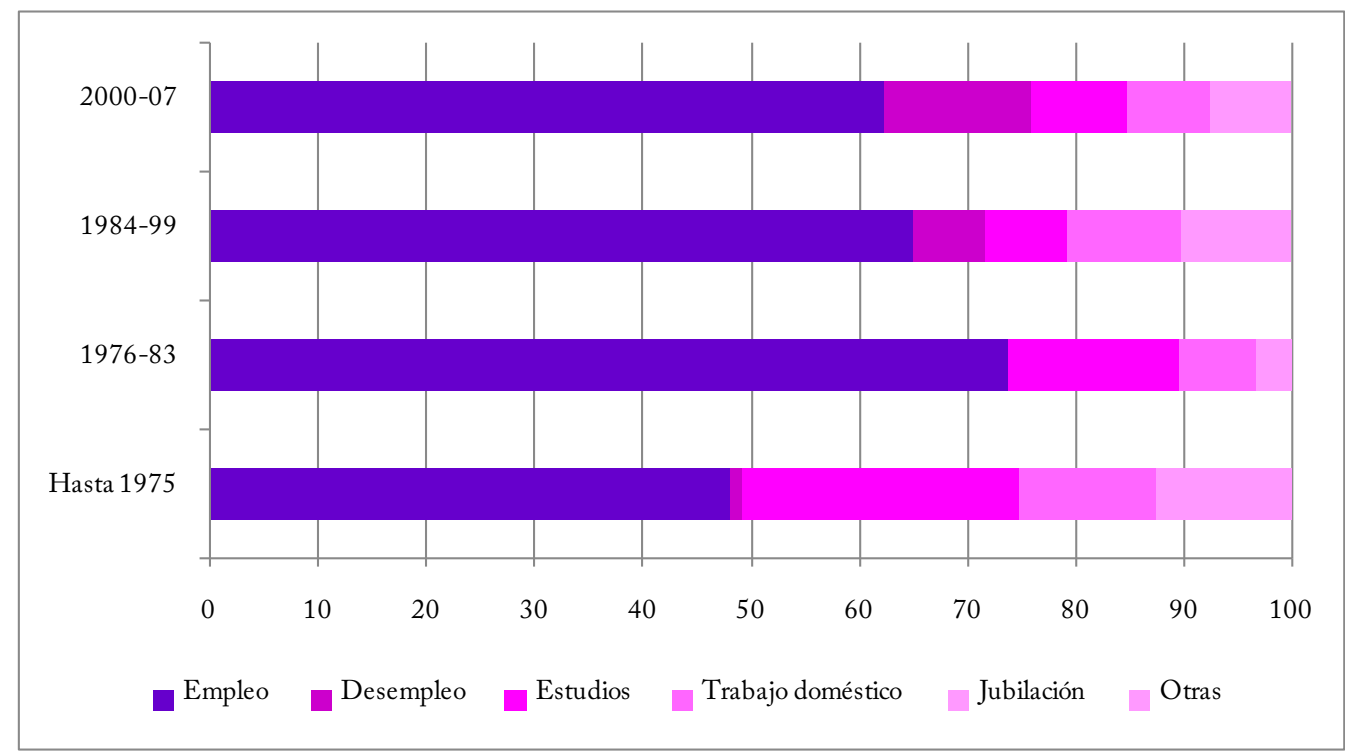

Fuente: Instituto Nacional de Estadística de España (INE), Encuesta Nacional de Inmigrantes (ENI) y elaboración propia.

ción de activos (72 y 76\% respectivamente), pero a diferencia del flujo anterior contaron con una creciente participación de desempleados: 9,4\% en el período $1984-1999$ y $13,6 \%$ en el período 2000-2007. Dicha participación fue consecuencia del incremento del desempleo en Argentina a raíz de las crisis económicas de 1989 y 2001, sobre todo de esta última, porque afectó de forma muy negativa al empleo (según el Censo de 2001 la tasa de desempleo trepó al $28,5 \%) .{ }^{74}$ La proporción de estudiantes

74. Debe considerarse que la población desocupada estimada por una y otra fuente son universos diferentes. Para la ENI son personas emigradas a España entre los 16 y 64 años y que se encontraban en ese país en enero de 2007. Para el Censo Nacional de Población, Hogares se mantuvo más o menos constante (7\% y 9\%) en ambos flujos, aunque fue bastante inferior a la del flujo del exilio; en cambio las personas que se dedicaban solo a labores domésticas se mantuvieron en valores similares en los tres períodos: oscilaron entre el 10\% y el $7 \%$ (véase, Gráfico 1).

En la medida que una gran parte de los emigrados era económicamente activos en $\mathrm{Ar}$ gentina, es clave saber más sobre su actividad. Atendiendo a la situación profesional, consta-

y Viviendas 2001 (INDEC, Argentina) se trata de personas de 14 y más años entrevistadas en la fecha de referencia. 
Tabla 3. España. Población nacida en Argentina, emigrada entre los 16 y 64 años, según situación profesional en el último empleo en Argentina antes de emigrar y sexo. A 1 de enero de 2007. En porcentajes

\begin{tabular}{|c|c|c|c|c|c|}
\hline \multirow{2}{*}{ Sexo } & \multirow{2}{*}{ Situación profesional } & \multicolumn{3}{|c|}{ Periodo de llegada } & \multirow[b]{2}{*}{ Total } \\
\hline & & 1976-83 & 1984-99 & 2000-07 & \\
\hline \multirow{7}{*}{ Hombre } & Asalariado sector público & 52,6 & 12,1 & 7,6 & 10,8 \\
\hline & Asalariado sector privado & 15,7 & 51,3 & 73,0 & 63,6 \\
\hline & Empresario sin asalariados & 10,6 & 21,7 & 12,0 & 15,6 \\
\hline & Empresario con asalariados & 13,7 & 7,6 & 2,9 & 4,7 \\
\hline & Miembro de una cooperativa & 0,0 & 0,5 & 0,0 & 0,2 \\
\hline & Ayuda familiar & 0,0 & 1,7 & 2,0 & 1,8 \\
\hline & Otra situación & 7,5 & 5,0 & 2,5 & 3,4 \\
\hline \multirow{7}{*}{ Mujer } & Asalariado sector público & 7,2 & 15,8 & 21,6 & 19,2 \\
\hline & Asalariado sector privado & 86,3 & 54,2 & 57,2 & 57,8 \\
\hline & Empresario sin asalariados & 0,0 & 17,4 & 15,3 & 15,5 \\
\hline & Empresario con asalariados & 0,0 & 8,9 & 4,3 & 5,2 \\
\hline & Miembro de una cooperativa & 0,0 & 0,0 & 0,0 & 0,0 \\
\hline & Ayuda familiar & 6,5 & 2,6 & 0,3 & 1,1 \\
\hline & Otra situación & 0,0 & 1,2 & 1,4 & 1,2 \\
\hline
\end{tabular}

Fuente: Instituto Nacional de Estadística de España (INE), Encuesta Nacional de Inmigrantes (ENI) y elaboración propia.

tamos que la amplia mayoría de los emigrados trabajaba en Argentina en relación de dependencia (74\% de los hombres y $77 \%$ de las mujeres). Las mujeres estaban sobrerepresentadas en el sector público (19,2\% frente al 10,8\%) y los hombres en el privado (63,6\% frente al 57,8\%) (véase, Tabla 3).

De acuerdo al período de llegada, hallamos en primer lugar una clara distinción entre el flujo del exilio y los flujos posteriores en cuanto a la participación de asalariados del sector público: $36 \%$ en el primero y en torno al $14 \%$ en los siguientes. Dos cuestiones explican estos datos: la represión en los lugares de trabajo durante la dictadura militar (cesantías, purgas ideológi- cas y miedo generalizado) y el deterioro de las condiciones de trabajo en la función pública. En segundo término, se aprecia una disminución de cuentapropistas en el "flujo del corralito" (2000 a 2007) respecto a los emigrados en el flujo anterior: del $28 \%$ al $17 \%$. En la medida que la proporción de cuentapropistas en $\mathrm{Ar}$ gentina se mantuvo en proporciones similares entre 1991 y $2010^{75}$, no es posible explicar este hecho con los datos disponibles.

75. De acuerdo con los Censos de Población y Vivienda, la proporción de cuentapropistas sobre el total de la población ocupada de 14 y más años era de $23 \%$ en 1991 , $20 \%$ en 2001 y $18,5 \%$ en 2010. 
Gráfico 2. España. Población nacida en Argentina, emigrada entre los 16 y 64 años, según situación sociolaboral en el último empleo en Argentina antes de emigrar y período de llegada. A 1 de enero de 2007. En porcentajes

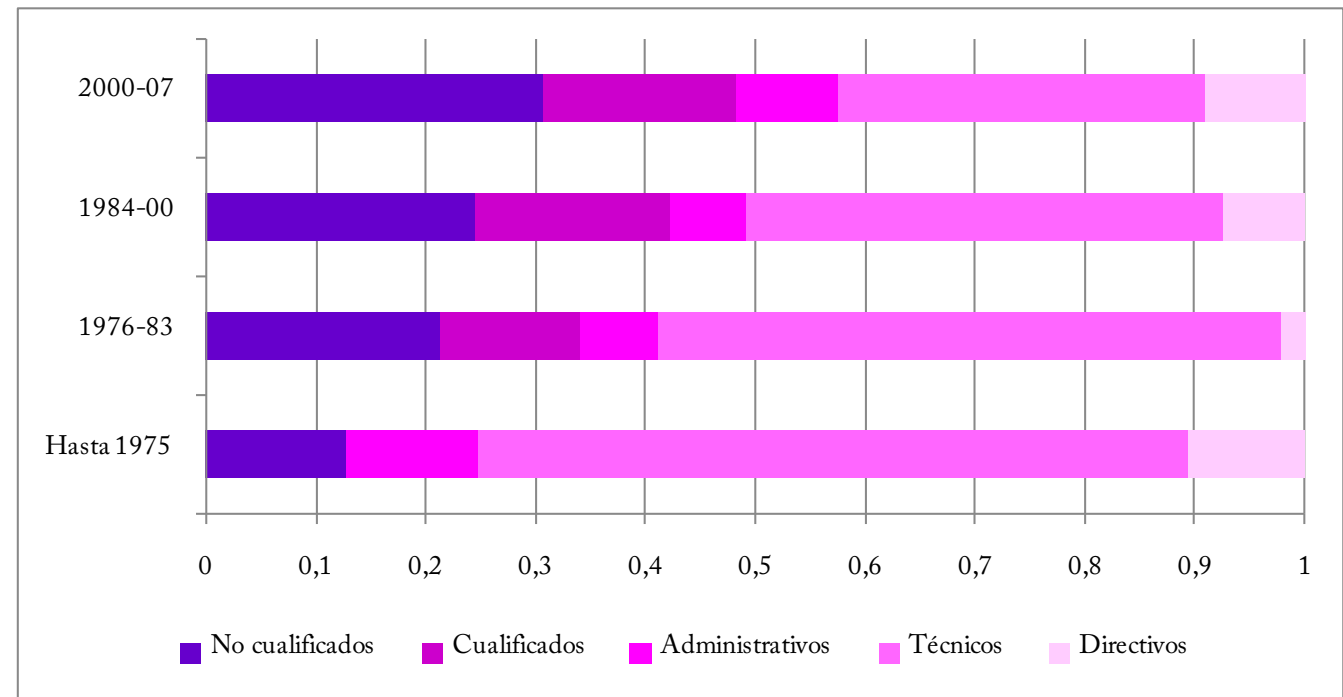

Fuente: Instituto Nacional de Estadística de España (INE), Encuesta Nacional de Inmigrantes (ENI) y elaboración propia.

En cuanto a los perfiles sociolaborales de los inmigrantes en su último empleo en Argentina, en el Gráfico 2 puede observarse que fueron variando con el trascurso del tiempo: descendió la proporción de directivos y técnicos-profesionales (del 47\% entre 1976-1983 al $14 \%$ en el período 2000-2007), mientras aumentó la de trabajadores manuales no cualificados (desde un $26 \%$ entre los emigrados entre 1976 y 1983 hasta el 60\% entre los emigrados en el período 2000-2007). En cambio, quienes realizaban tareas manuales cualificadas y administrativas no variaron de un flujo a otro (se mantuvieron en torno al 16\% los primeros y al 10\% los segundos). Esta evolución pone en evidencia que disminuyó de forma progresiva el nivel de selectividad en la emigración de argentinos a España en las últimas décadas. Una tendencia que se explica, sobre todo, por la evolución de la demanda en el mercado de trabajo español debido a la necesidad creciente de cubrir puestos de trabajo menos cualificados, más que por la evolución del estatus sociolaboral de los trabajadores en Argentina, que ha variado poco desde $1991 .{ }^{76}$

76. En Argentina, según el Censo Nacional de Población y Vivienda 1991, la distribución de la población ocupada de 14 años y más, de acuerdo a su calificación ocupacional, era la siguiente: científico-profesional $5 \%$; técnica $16 \%$; operativa $40 \%$, no calificada $27 \%$; $\mathrm{n} / \mathrm{s} 4 \%$. En el año 2003 (primer trimestre), la Encuesta Permanente de Hogares estimó, para la misma población, una distribución con valores similares: científicoprofesional $9 \%$; técnica $15 \%$; operativa $49 \%$, no calificada $26 \%, \mathrm{n} / \mathrm{s} 1 \%$. 
En la Tabla 4 presentamos datos sobre la duración del contrato o relación laboral que tenían los inmigrantes en Argentina antes del desplazamiento. ${ }^{77}$ Allí observamos que alrededor del 38\% de los inmigrantes argentinos que trabajaron como asalariados antes de emigrar de Argentina tuvieron una relación laboral de carácter temporal. La tasa de temporalidad de los hombres fue similar en los períodos 19841999 y 2000-2007; sin embargo, las mujeres arribadas en el último período tuvieron un nivel de temporalidad más elevado que el de aquellas emigradas en el flujo anterior (40\% frente a $25 \%$, respectivamente). La combinación de flexibilización laboral y discriminación de género en el mercado de trabajo explican esta situación

En síntesis, en la población emigrada a España existió una notable selectividad a favor de la población activa en Argentina, sobre todo entre las mujeres, aunque las emigradas presentaban niveles de inactividad relativamente altos debido al desigual reparto de las tareas reproductivas. Los datos relativos al último empleo en Argentina ponen de manifiesto la existencia de una cantidad significativa de población ocupada en empleos técnicos y administrativos (46\%), pero también en trabajos manuales (45\%). La mitad de las mujeres se encontraba en el primer grupo y la mitad de los hombres en el segundo. Puede decirse, entonces, que se trató de una emigración con orígenes sociales diversos, característica que ya había sido seña-

77. En este caso conviene relativizar los datos relativos a flujos anteriores a 1983 porque, los contratos temporales y otros mecanismos de flexibilización laboral se generalizaron en Argentina durante la década de 1990. Un análisis del tema puede verse en: ALTIMIR, Oscar y BECCARIA, Luis, "El mercado de trabajo bajo el nuevo régimen económico en Argentina" CEPAL. Series Reformas Económicas, № 28, 1999.

64 lada en otros estudios. ${ }^{78}$ En términos de flujos, en los dos más recientes de carácter económico emigró más de un $40 \%$ de trabajadores manuales, en el último esta proporción alcanzó el 50\%, mientras en el flujo del exilio más de dos tercios de los emigrados que se encontraban ocupados antes del desplazamiento desempeñaban tareas técnicas y administrativas. Esta tendencia vuelve a poner de manifiesto la particularidad del exilio, en el marco de las migraciones de argentinos a España, al tiempo que demuestra una paulatina disminución del nivel de selectividad en esta emigración.

\section{La situación en el primer empleo en España}

Si observamos ahora la situación profesional de los argentinos en el primer empleo en España hallamos que la proporción de asalariados del sector público y de empresarios disminuyó con el tiempo de residencia ${ }^{79} \mathrm{y}$, paralelamente, aumentaron los asalariados del sector privado (véase, Tabla 5). Es decir, los flujos más antiguos estaban más representados en las situaciones profesionales más ventajosas. Por ejemplo, en el flujo del exilio, los empleados

78. ACTIS, Walter; ESTEBAN, Fernando Osvaldo, “Argentinos en España...”, Op. Cit.; JIMÉNEZ, Cecilia, Desclasamiento y..., Op. Cit.

79. La escasa representación de empresarios en el flujo del exilio puede deberse a un sesgo estadístico pero también a un hecho social. En aquel momento se produjo un aumento de la oferta de puestos de trabajo, cualificados y semicualificados, en grandes empresas del sector privado en proceso de expansión y modernización (energía, telecomunicaciones, banca, seguros). Éstos, podían significar una alternativa mejor que emprendimientos por cuenta propia en términos de estabilidad y oportunidades de promoción. 
Tabla 4. España. Población nacida en Argentina, emigrada entre los 16 y 64 años, según duración del contrato o acuerdo laboral en el último empleo en Argentina, sexo y período de llegada. A 1 de enero de 2007. En porcentajes

\begin{tabular}{llcccc}
\hline \multirow{2}{*}{ Sexo } & \multirow{2}{*}{ Contrato } & \multicolumn{3}{c}{ Período de llegada } & \multirow{2}{*}{ Total } \\
\cline { 3 - 5 } & & $\mathbf{1 9 7 6 - 8 3}$ & $\mathbf{1 9 8 4 - 9 9}$ & $\mathbf{2 0 0 0 - 0 7}$ & \\
\hline \multirow{2}{*}{ Hombre } & Indefinida & 49,6 & 64,0 & 61,5 & 61,5 \\
& Temporal & 50,4 & 36,0 & 38,5 & 38,5 \\
\multirow{2}{*}{ Mujer } & Indefinida & 40,4 & 75,1 & 59,5 & 62,5 \\
& Temporal & 59,6 & 24,9 & 40,5 & 37,5 \\
\hline
\end{tabular}

Fuente: Instituto Nacional de Estadística de España (INE), Encuesta Nacional de Inmigrantes (ENI) y elaboración propia.

públicos alcanzaron el 10\% mientras representaron el 6\% entre los emigrados en el período $1984-1999$ y apenas superaron el 3\% entre los arribados durante 2000 - 2007. Asimismo, la proporción de empresarios en el flujo emigrado en el período 1984-1999 más que duplicó a la del flujo del corralito (17\% versus $5 \%$ en el caso de los empresarios sin asalariados y $2 \%$ frente a ninguno en el de aquellos que tenían empleados a cargo).

No obstante, esta conclusión debe matizarse ya que los empleos en el sector público no son, necesariamente, mejores que los empleos en el sector privado, ni el trabajo por cuenta propia significa siempre una situación más favorable que el trabajo por cuenta ajena. Además, la posibilidad de comenzar la actividad laboral en España en situación de empresario puede demandar un capital inicial que depende, muchas veces, de las condiciones de partida del país de origen, condiciones que hacen referencia a la clase social de los inmigrantes pero también a constreñimientos macroeconómicos coyunturales (por ejemplo, en el caso de Argentina, la hiperinflación, el Plan Bonex, el "corralito" financiero, la pesificación forzosa).

La situación profesional de las mujeres argentinas en el primer empleo en España fue singularmente diferente a la de los hombres debido a una mayor participación en el sector público y en el empresariado, este último, sobre todo en carácter de trabajadora autónoma (véase, Tabla 5). Esta situación se percibe en todos los flujos migratorios y era más significativa a medida que aumentaba el tiempo de residencia. Así, entre los emigrados en el período 2000-2007 hubo dos veces más mujeres que hombres asalariados en el sector público $(4,1 \%$ y $2,1 \%$ respectivamente), mientras en los inmigrantes que llegaron entre 1976 y 1983 esa diferencia se multiplicó por cinco (20,8\% frente a 4,5\%). Asimismo, la proporción de empresarios era del $9 \%$ entre las mujeres emigradas en el flujo del corralito, frente al 1,5\% de los hombres; y era del $13 \%$ en el flujo del exilio frente al $2 \%$ de hombres.

Las causas de este fenómeno obedecen a dos patrones de inserción laboral de la mujer en 
Tabla 5. España. Población nacida en Argentina, emigrada entre los 16 y 64 años, según situación profesional en el primer empleo en España, sexo y período de llegada. A 1 de enero de 2007. En porcentajes

\begin{tabular}{clcccc}
\hline \multirow{2}{*}{ Sexo } & \multirow{2}{*}{ Situación profesional } & \multicolumn{3}{c}{ Período de llegada } & \multirow{2}{*}{ Total } \\
\cline { 3 - 5 } & & $\mathbf{1 9 7 6 - 8 3}$ & $\mathbf{1 9 8 4 - 9 9}$ & $\mathbf{2 0 0 0 - 0 7}$ & \\
\hline \multirow{3}{*}{ Hombre } & Asalariado sector público & 4,5 & 3,5 & 2,1 & 2,6 \\
& Asalariado sector privado & 93,5 & 77,2 & 93,4 & 87,4 \\
\cline { 2 - 5 } & Empresario sin asalariados & 2,0 & 15,9 & 1,5 & 6,8 \\
\cline { 2 - 5 } & Empresario con asalariados & 0,0 & 3,3 & 0,0 & 1,0 \\
\cline { 2 - 5 } & Ayuda familiar & 0,0 & 0,0 & 1,0 & 1,1 \\
& Otra situación & 0,0 & 0,0 & 2,0 & 1,2 \\
& Asalariado sector público & 20,8 & 9,9 & 4,1 & 6,2 \\
& Asalariado sector privado & 66,1 & 66,8 & 81,2 & 76,6 \\
\cline { 2 - 5 } Mujer & Empresario sin asalariados & 13,0 & 20,2 & 9,3 & 12,7 \\
& Ayuda familiar & 0,0 & 3,1 & 1,0 & 1,4 \\
& Otra situación & 0,0 & 0,0 & 4,4 & 3,1 \\
\hline
\end{tabular}

Fuente: Instituto Nacional de Estadística de España (INE), Encuesta Nacional de Inmigrantes (ENI) y elaboración propia.

España. Uno más habitual en mujeres autóctonas que consiste en acceder a puestos de trabajo cualificados en el sector público y en grandes empresas privadas. En este caso, los concursos de méritos permiten salvar la discriminación por género que habitualmente impera en el mercado de trabajo. ${ }^{80}$ Otro patrón de inserción laboral, más típico en las inmigrantes extracomunitarias, fue el trabajo en hogares (servicio doméstico, cuidado de niños, enfermos y ancianos) realizando aportes a la Seguridad Social en carácter de trabajadoras

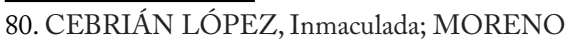
RAYMUNDO, Gloria. "La situación de las mujeres... Op. Cit.

66 autónomas (aunque siempre ha sido un sector con elevados índices de irregularidad). Esta actividad fue uno de los "puertos de entrada" al mercado de trabajo español más transitado por las mujeres extranjeras. ${ }^{81}$ Sin embargo, algunos colectivos, como el argentino, también contaron con un volumen elevado de mujeres que comenzaron la actividad económica en España desarrollando actividades empresariales en el sector servicios (hostelería, comercio al por menor, textil). En este caso, el "negocio propio" fue una estrategia de refugio para no caer en el servicio doméstico, una continuidad profe-

81. PARELLA, Sonia, Mujer, inmigrante, trabajadora. La triple discriminación, Anthropos, Barcelona, 2003. 
sional de la trayectoria ocupacional anterior o un proyecto empresarial que vinculaba a toda la familia. ${ }^{82}$

Atendiendo al estatus sociolaboral en el primer empleo en España, en el Gráfico 3 se puede percibir que fue claramente distinto de acuerdo al período de llegada, y con una tendencia similar a la señalada en el análisis de la situación profesional: los inmigrantes más recientes ingresaron al mercado de trabajo en una situación menos favorable que aquellos que tenían más tiempo de residencia en España. En este caso, ello se percibe a través del aumento de trabajadores manuales no cualificados y del descenso de técnicos y directivos. Entre los argentinos que emigraron entre 1975 y 1983 , el 26\% desempeñaron actividades manuales no cualificadas en su primer empleo, mientras ese porcentaje aumentó al 41\% entre los que llegaron en el período 1984-1999 y, finalmente, fueron más de la mitad de los integrantes del flujo del corralito (51\%). Una tendencia similar muestra la inserción de trabajadores manuales cualificados. Por el contrario, descendió significativamente la proporción de aquellos que ingresaron al mercado de trabajo en las categorías superiores: los directivos eran el 3,2\% entre los arribados en 1984-1999 y 1,2\% en el flujo del período 2000-2007; en la categoría de técnicos y profesionales el descenso fue de 30 puntos porcentuales (del 47\% al 12\%) desde 1976-1983 a 2000-2007.

Los elevados índices de empleo temporal son una de las características diferenciales del empleo en España, dentro de las economías desa-

82. OSO CASAS, Laura; VILLARES VARELA, María, "Mujeres inmigrantes lationamericanas y empresariado étnico: dominicanas en Madrid, argentinas y venezolanas en Galicia", en Revista Galega de Economia, Santiago de Compostela, 2005, V. 14, No. 1 y 2, pp. 1-19. rrolladas. La tasa de temporalidad (proporción de temporales sobre el total de asalariados) se mantuvo en torno al 30\% desde 1989 y sólo descendió a partir de 2008 (para ubicarse alrededor del 25\%), a consecuencia de la fuerte destrucción de empleo temporal que ocasionó la crisis económica. En dicho contexto, los trabajadores de origen extranjero presentaron tasas de temporalidad que duplicaron a la de trabajadores autóctonos. Precisamente, los datos recogidos por la ENI indican que, excepto los hombres nacidos en Francia y las mujeres de Alemania, todos los grupos superaron el $50 \%$ de temporalidad en el primer empleo en España. ${ }^{83}$ Los argentinos, como se aprecia en la Tabla 6, tuvieron tasas superiores al $60 \% \sin$ distinción significativa según el período de llegada y el sexo (excepto las mujeres arribadas entre 1983 y 1999 que tuvieron una tasa notablemente inferior). Este indicador sugiere que, aunque los inmigrantes más antiguos tuvieron una inserción laboral inicial más favorable que los más recientes la precariedad laboral fue un denominador común entre los inmigrantes argentinos.

Una explicación de este nivel de temporalidad y del diferencial con los trabajadores españoles viene dada porque los sectores económicos donde se concentraron los inmigrantes fueron los que tenían mayores tasas de temporalidad (construcción, hostelería y hogares que emplean personal doméstico), aunque también en cada uno de estos sectores los inmigrantes tuvieron tasas superiores a las de los españoles. Desde esta perspectiva, la menor tasa de temporalidad de las mujeres argentinas emigradas entre 1983 y 1999 se explicaría a partir de su

83. COLECTIVO IOE y FERNÁNDEZ, Mercedes, Encuesta Nacional de Inmigrantes 2007: el Mercado de trabajo y las redes sociales de los inmigrantes, Ministerio de Trabajo e Inmigración, Madrid, 2010. 
Grafico 3. España. Población nacida en Argentina, emigrada entre los 16 y 64 años, según situación sociolaboral en el primer empleo en España y período de llegada. A 1 de enero de 2007. En porcentajes.

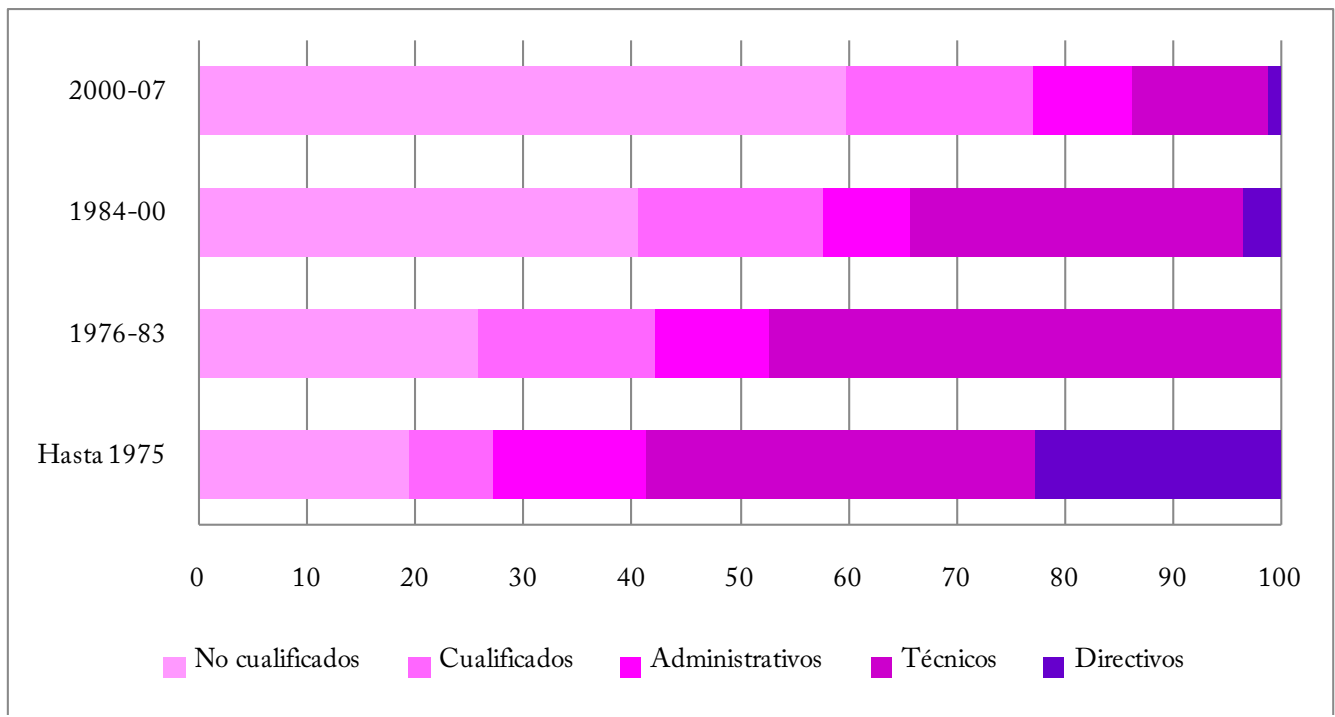

Fuente: Instituto Nacional de Estadística de España (INE), Encuesta Nacional de Inmigrantes (ENI) y elaboración propia.

menor participación en el servicio doméstico ( $11 \%$ frente al $18 \%$ de las mujeres migradas en 1976-1983 y 2000-2007).

\section{Conclusiones}

En la población emigrada a España entre 1975 y 2007 existió una notable selectividad a favor de la población activa en Argentina, sobre todo entre las mujeres. Los datos relativos al último empleo en Argentina ponen de manifiesto la existencia de una cantidad significativa de población ocupada en empleos técnicos y administrativos (46\%), pero también en trabajos manuales (45\%). La mitad de las mujeres se encontraba en el primer grupo y la mitad de los hombres en el segundo. Puede decirse, entonces, que se trató de una emigración con orígenes sociales diversos, característica que ya había sido señalada en estudios previos. ${ }^{84}$

A partir de la explotación de datos estadísticos sobre relación de dependencia, estatus ocupacional y duración del contrato en el primer en empleo en España, contrastamos empíricamente la hipótesis de referencia en el campo, según la cual la inserción laboral inicial de los inmigrantes se produce en categorías inferiores a las que tenían en sus países de origen. El

84. ACTIS, Walter; ESTEBAN, Fernando Osvaldo, “Argentinos en España...”, Op. Cit.; JIMÉNEZ, Cecilia, Desclasamiento y..., Op. Cit. 
Tabla 6. España. Población nacida en Argentina, emigrada entre los 16 y 64 años, según duración del contrato o acuerdo laboral en el primer empleo en España, sexo y período de llegada. A 1 de enero de 2007. En porcentajes

\begin{tabular}{clcccc}
\hline Sexo & $\begin{array}{c}\text { Duración del } \\
\text { contrato }\end{array}$ & $\mathbf{1 9 7 6 - 8 3}$ & $\mathbf{1 9 8 4 - 9 9}$ & $\mathbf{2 0 0 0 - 0 7}$ & Total \\
\hline \multirow{3}{*}{ Hombre } & No sabe & 10,9 & 7,2 & 6,5 & 6,7 \\
& Indefinida & 26,7 & 24,9 & 26,7 & 28,9 \\
& Temporal & 62,5 & 67,9 & 66,9 & 64,4 \\
& No sabe & 9,5 & 7,0 & 10,5 & 9,5 \\
\multirow{3}{*}{ Mujer } & Indefinida & 21,0 & 32,7 & 20,4 & 23,5 \\
& Temporal & 69,5 & 60,4 & 69,1 & 67,1 \\
\hline
\end{tabular}

Fuente: Instituto Nacional de Estadística de España (INE), Encuesta Nacional de Inmigrantes (ENI) y elaboración propia.

análisis ha confirmado la hipótesis. Se ha demostrado que la emigración produjo inmediatamente un proceso de movilidad ocupacional descendente que se puso de manifiesto a través de un incrementó de trabajadores asalariados en menoscabo de autónomos y empresarios, en una significativa disminución de estatus ocupacional y en el empeoramiento de la seguridad en el empleo.

En la Tabla 7 puede advertirse que el 20\% de los inmigrantes experimentó un empeoramiento de la situación profesional respecto a la que tenía en Argentina, el 48\% lo hizo respecto a la situación sociolaboral y el $44 \%$ a la seguridad en el empleo. Si se tiene en cuenta, además, que en el último empleo en Argentina el $68 \%$ eran asalariados, $20 \%$ no podían descender porque ya ocupaban la categoría de trabajadores no cualificados y $31 \%$ tenían contratos temporales, puede afirmarse que la emigración produjo una movilidad ocupacional descendente muy significativa. Ésta produjo cierta igualación "por abajo" entre hombres y mujeres estrechando las diferencias que existían en el último empleo en Argentina a favor de los hombres.

La segunda hipótesis sostiene que los flujos migratorios más antiguos, particularmente el exilio, ingresaron al mercado de trabajo español en mejores condiciones que los más recientes de carácter predominantemente económico. En este caso, el análisis de los datos confirma la hipótesis sólo parcialmente. Como puede observarse en la Tabla 8, no se aprecian diferencias contundentes entre el flujo del exilio y los posteriores flujos económicos en la situación profesional y la seguridad en el empleo de los migrantes. En cambio, se comprueba una mejor situación respecto al estatus ocupacional de los emigrados en el flujo del exilio frente a los emigrados en el "flujo de la hiperinflación" y en el "flujo del corralito": la proporción de trabajadores manuales era significativamente 
Tabla 7. España. Población nacida en Argentina, emigrada entre los 16 y 64 años, según mejoró, empeoró o mantuvo sus condiciones de trabajo desde el último empleo en Argentina al primero en España. A 1 de enero de 2007

\begin{tabular}{lccc}
\hline \multirow{2}{*}{ Condiciones de trabajo } & \multicolumn{3}{c}{$\begin{array}{c}\text { Desde el último empleo en Argentina al } \\
\text { primero en España }\end{array}$} \\
\cline { 2 - 4 } & Mejor & Igual & Peor \\
\hline Situación profesional & $8 \%$ & $72 \%$ & $20 \%$ \\
Situación sociolaboral & $12 \%$ & $40 \%$ & $48 \%$ \\
Seguridad en el empleo & $4 \%$ & $51 \%$ & $44 \%$ \\
\hline
\end{tabular}

Fuente: Instituto Nacional de Estadística de España (INE), Encuesta Nacional de Inmigrantes (ENI) y elaboración propia.

menor en el primero (42\%) que en los dos siguientes ( $58 \%$ y $77 \%$ respectivamente).

La mejor inserción laboral de los exiliados frente a los migrantes económicos que llegaron posteriormente (y entre éstos, quienes emigraron en los años ' 80 y '90 frente a aquellos que lo hicieron en la primera década de 2000) no puede explicarse a raíz de diferencias en la composición demográfica o en las dotaciones de capital humano. Según la ENI, no existían diferencias significativas entre los flujos respecto al sexo, la edad y los arreglos familiares de los migrantes. Asimismo, al nivel educativo de los argentinos al arribar a España también era similar en los distintos flujos. Además, aunque los migrantes económicos tenían en conjunto más experiencia laboral, debido a una proporción más elevada de personas económicamente activas, el flujo del exilio partía con ventaja porque tenía una mejor inserción laboral previa en Argentina.
Por consiguiente, una explicación plausible de las inserciones laborales diferenciadas de exiliados y migrantes económicos sobre la que habrá que continuar investigando puede hallarse en los contextos de recepción que enfrentó cada flujo. En particular, tres elementos de esos contextos relacionados entre sí, ya destacados en la literatura: ${ }^{85}$ la política migratoria, el volumen y la composición de la inmigración y la estructura del mercado de trabajo. En este sentido, el flujo del exilio arribó a España cuando la política migratoria estaba enfocada aún en la regulación de la emigración internacional de españoles y no existían restricciones severas para residir y trabajar legalmente en el país. La inmigración extranjera estaba compuesta sobre todo por europeos, retirados de la actividad económica o empleados en empresas de sus países de origen, y por exiliados políticos sudamericanos. En ambos casos, era una migración socialmente invisible por su escaso

85. Cachón, Lorenzo, Marco institucional..., Op. Cit.; Herranz, Yolanda, "La inmigración latinoamericana...", Op. Cit. 
Tabla 8. España. Población nacida en Argentina, emigrada entre los 16 y 64 años, según situación profesional, situación sociolaboral, seguridad en el empleo y período de llegada. A 1 de enero de 2007.

\begin{tabular}{lcccccc}
\hline & \multicolumn{2}{c}{ Último empleo en Argentina } & \multicolumn{3}{c}{ Primer empleo en España } \\
\hline & $1976-83$ & $1984-00$ & $2000-07$ & $1976-83$ & $1984-00$ & $2000-07$ \\
\hline \multicolumn{7}{c}{ Situación profesional } \\
\hline Asalariado & $74 \%$ & $68 \%$ & $84 \%$ & $94 \%$ & $80 \%$ & $94 \%$ \\
Empresario & $26 \%$ & $32 \%$ & $16 \%$ & $6 \%$ & $20 \%$ & $6 \%$ \\
\hline Manual & \multicolumn{7}{c}{ Situación sociolaboral } \\
Administ.-Tecnico & $64 \%$ & $52 \%$ & $48 \%$ & $42 \%$ & $58 \%$ & $77 \%$ \\
Directivo & $2 \%$ & $8 \%$ & $9 \%$ & $58 \%$ & $39 \%$ & $22 \%$ \\
& \multicolumn{7}{c}{ Seguridad en el empleo } \\
\hline Contrato indefinido & $46 \%$ & $69 \%$ & $62 \%$ & $24 \%$ & $28 \%$ & $24 \%$ \\
Contrato temporal & $54 \%$ & $31 \%$ & $38 \%$ & $76 \%$ & $72 \%$ & $76 \%$ \\
\hline
\end{tabular}

Fuente: Fuente: Instituto Nacional de Estadística de España (INE), Encuesta Nacional de Inmigrantes (ENI) y elaboración propia.

volumen y similitud con la población autóctona. La dinámica del mercado de trabajo estaba caracterizada por una gran movilidad ocupacional hacia los servicios avanzados, tanto en el sector público por la nueva organización territorial del Estado y la creación del Estado de Bienestar, como en el sector privado por la internacionalización de las grandes empresas privatizadas (telecomunicaciones, bancos, energéticas, transporte). Los exiliados argentinos se vieron beneficiados por esta coyuntura y se insertaron en las posiciones más altas de la estructura ocupacional (empleos de cuello blanco).

En cambio, los migrantes económicos que arribaron a partir de mediados de la década de 1980 (después de la entrada en vigor de la L.O.
7/1985) hallaron un contexto de recepción cada vez más hostil. La política migratoria, $y$ las políticas sociales en general (sobre todo las relativas al empleo), discriminaron entre ciudadanos de países de la Unión Europea y de terceros países asignando derechos y obligaciones diferentes, siendo éste último el colectivo más perjudicado. Al mismo tiempo, España se fue convirtiendo en uno de los mayores receptores mundiales de inmigración extranjera, atraída por el crecimiento de la economía y del bienestar social. La base de ese crecimiento fue un modelo de desarrollo de baja productividad e intensivo en mano de obra, con la construcción, el turismo y los servicios a las empresas como sectores más pujantes. Con este modelo se fue conformando una estructura productiva etnofragmentada en la cual los inmigran- 
tes extracomunitarios ocuparon empleos en el segmento secundario del mercado de trabajo. Empleos cada vez más disputados por una oferta creciente de mano de obra extranjera previamente dispuesta en situación de vulnerabilidad por el Estado a través del marco jurídico que regula la extranjería. Se trataba de puestos vacantes por la propia dinámica demográfica (reducción de la población activa autóctona a raíz de la disminución de la tasa de natalidad y de la mayor permanencia de los jóvenes en el sistema educativo), pero también por una dinámica social: el incremento de las expectativas sociales de los trabajadores españoles que, gracias a la mejora de sus condiciones materiales de existencia, les condujo a permanecer inactivos o desocupados (formándose o no) hasta hallar el empleo deseado.<smiles>C[As]</smiles> 


\section{Bibliografía}

ACTIS, Walter; ESTEBAN, Fernando Osvaldo, "Argentinos en España: inmigrantes a pesar de todo", en Migraciones, Madrid, 2008, No 23, pp. 79-115.

AJA, Eliseo, "La evolución sobre normativa sobre inmigración", en AJA, Eliseo y ARANGO, Joaquín (editores) Veinte años de inmigración en España. Perspectivas jurídica y sociológica (1985-2005), Fundació CIDOB, Barcelona, 2006, pp. 17-44.

AKKAYMAK, Guliz, "A Bourdieuian Analysis of Job Search Experiences of Immigrants in Canada”, en Migration E Integration, 2017, № 18, pp. 657-674.

ALONSO, Luis Enrique, Trabajo y ciudadanía: estudios sobre la crisis de la sociedad salarial, Trotta, Madrid, 1999.

ALONSO, Luis E., "Ciudadanía, sociedad del trabajo y Estado de Bienestar: los derechos sociales en la era de la fragmentación”, en PÉREZ LEDESMA, Manuel (compilador) Ciudadanía y democracia, Pablo Iglesias, Madrid, 2000, pp. 159-192.

ALTIMIR, Oscar y BECCARIA, Luis, "El mercado de trabajo bajo el nuevo régimen económico en Argentina”, en CEPAL. Series Reformas Económicas, 1999, № 28.

ANGUIANO TÉLLEZ, María Eugenia, "Emigración reciente de latinoamericanos a España: trayectorias laborales y movilidad ocupacional”, en Papeles de población, Toluca-México, 2002, V. 33, No 8, pp. 101115 .

AYSA-LASTRA, María y CACHÓN, Lorenzo, "Movilidad ocupacional segmentada: el caso de los inmigrantes no comunitarios en España”, en Revista Española de Investigaciones Sociológicas, Madrid, 2013, No 144, pp.23-47.

BAUER, Thomas y ZIMMERMANN, Klaus F., “Occupational mobility of ethnic migrants”, en IZA Discussion Paper, Bonn, No 58, 1999, pp. 1-24.

BECKER, Gary, "Investment in Human Capital: a Theoretical Analysis”, en The Journal of Political Economy, Chicago, 1962, V. 70, No 5, pp. 9-49.

BAKER, Michael y BENJAMIN, Dwayne, "The role of the family in immigrants' labor-market activity: An evaluation of alternative explanations", en American Economic Review, Nashville, 1997, V. 87, No 4, pp. 705-727.

BLAUG, Mark, “The Empirical Status of Human Capital Theory: A Slightly Jaundiced Survey”, en Journal of Economic Literature, Pittsburgh, 1976, No 14, V. III, pp. 827-855.

BRAVERMAN, Harry, Trabajo y capital monopolista, Siglo XXI, Madrid, 1975.

CACHÓN, Lorenzo, "Marco institucional de la discriminación y tipos de inmigrantes en el mercado de trabajo en España”, en Revista Española de Investigaciones Sociológicas, Madrid, 1995, No 69, pp. 105-124. 
CACHÓN, Lorenzo, La España inmigrante: marco discriminatorio, mercado de trabajo y politicas de integración, Anthropos, Barcelona, 2009.

CACOPARDO, María Cristina, MAGUID, Alicia, MARTÍNEZ, Rosana, "La nueva emigración de argentinos a España: el caso de los argentinos desde una perspectiva comparada", en Papeles de Población, Toluca-Méxio, 2007, № 51, pp. 9-44.

CEBRIÁN LÓPEZ, Inmaculada, MORENO RAYMUNDO, Gloria, "La situación de las mujeres en el mercado de trabajo español. Desajustes y retos", en Economía industrial, Madrid, 2008, No 367, pp. 121-137.

CERRUTI, Marcela y MAGUID, Alicia, "Familias divididas y cadenas globales de cuidados: la migración sudamericana a España”, en CEPAL. Serie Políticas Sociales, 2010, № 163.

CERRUTI, Marcela y MAGUID, Alicia, "Crisis económica en España y el retorno de inmigrantes sudamericanos”, en Migraciones Internacionales, 2016, No 8, V. III, pp. 155-189.

CHISWICK, Barry R., "A longitudinal analysis of the occupational mobility of immigrants", en DENNIS, Barbara (editora) Proceedings of the 30th annual winter meeting, Industrial Relations Research Association, 1977, Madison, WI IRRA, pp. 20-27

CHISWICK, Barry, "The effect of Americanization on the Earnings of Foreign-born Men", en The Journal of Political Economy, Chicago, 1978, V. 86, № 5, pp. 897-921.

CHISWICK, Barry. R., COHEN, Yinon y ZACH, Tzippi, "The labor market status of immigrants: Effects of the unemployment rate at arrival and duration of residence", en Industrial \& Labour Relations Review, Ithaca, 1997, V. 50, № 2, pp. 289-303.

CHISWICK, Barry, LEE, Yew Liang y MILLER, Paul, "A Longitudinal Analysis of Immigrant Occupational Mobility: A Test of the Immigrant Assimilation Hypotesis", en The International Migration Revierw, Staten Island, 2005, No 39, V. 2, pp. 332-353.

COLECTIVO IOE y FERNÁNDEZ, Mercedes, Encuesta Nacional de Inmigrantes 2007: el Mercado de trabajo y las redes sociales de los inmigrantes, Ministerio de Trabajo e Inmigración, Madrid, 2010.

DOERINGER, Peter y PIORE Michael, Internal labor markets and manpower adjustment, DC Heath and Company, New York, 1971.

DULEEP, Harriet y DOWHAN, Daniel J., "Revisiting the family investment model with longitudinal data the earnings growth of immigrant and U.S.-born women”, en IZA Discussion Paper, Bonn, 2002, № 568.

DULEEP, Harriet O. y REGETS, Mark C., "Immigrants and human-capital investment", en American Economic Review, Nashville, 1999, V. 89, No 2, pp. 186-191.

DULEEP, Harriet O. y SANDERS, Seth, "The decision to work by married immigrant women", en Industrial and Labor Relations Review, 1993, V. 46, No 4, pp. 677-690. 
ESTEBAN, Fernando Osvaldo, ACTIS, Walter, "Migración de personas cualificadas de Argentina a España ¿Una nueva fuga de cerebros?”, en LUCHILO, Lucas (coordinador) Más allá de la fuga de cerebros. Movilidad, migración y diásporas de argentinos calificados, Eudeba, Buenos Aires, 2011, pp. 117-168.

ESTEBAN, Fernando Osvaldo, "La inserción laboral de los inmigrantes argentinos en España. Un análisis a partir de la Encuesta Nacional de Inmigrantes 2007”, en Argumentos. Revista de Crítica Social, Buenos Aires, 2013, № 15, pp. 285-313.

ESTEBAN, Fernando Osvaldo, Cuatro décadas de migraciones de argentinos a España (1970-2010, Editorial Teseo, Buenos Aires, 2015.

FLIPPEN, Chenoa A., "Intersectionality at work: Determinants of labor supply among immigrant Hispanic women", en Population Association of American, 2013 annual meeting program, 2013. Disponible en: <http://paa2013.princeton.edu/abstracts/131053>.

FRIEDBERG, Rachel, "You Can't Take It with You? Immigrant Assimilation and the Portability of Human Capital”, en Journal of Labor Economics, Chicago, 2000, No 18, V. II, pp. 221-251.

FURTADO, Delia y THEODOROPOULOS, Nikolaos, "Why does intermarriage increase immigrant employment? The role of networks", en The B.E. Journal of Economic Analysis \& Policy, 2010, V.10, o 1, pp. 1-33.

GANDINI, Luciana, ${ }_{\dot{b}}$ Escapando de la crisis? Un estudio comparativo de trayectorias laborales de migrantes argentinos en la Ciudad de México y Madrid, UNAM-CRIM, Cuernavaca, 2015.

GARRIDO,Luis y GONZÁLEZ,Juan, "Mercado de trabajo, ocupación y clases sociales”,en GONZÁLEZ, Juan y REQUENA y DÍAZ DE REVENGA, Manuel (editores) Tres décadas de cambio social en España, Alianza, Madrid, 2005, pp. 81-126.

GOLDTHORPE, John H.; LLEWELLYN, C.; PAYNE, G., Class structure and social mobility in modern Britain, Oxford University Press, Oxford, 1980.

GRASSI, Estela, Politicas y problemas sociales en la sociedad neoliberal: la otra década infame (I), Espacio, Buenos Aires, 2003.

GRINBERG, León y GRINBERG, Rebeca, Psicoanálisis de la emigración y del exilio, Alianza, Madrid, 1982.

HEATH, Anthony y CHEUNG, Sin Yi, "The Comparative Study of Ethnic Minority Disadvantage", en Heath, Anthony y Cheung, Sin Yi (editores) Unequal chances. Ethnic Minorities in Western Labour Markets, Oxford University Press, Oxford, 2007, pp. 1-44.

HERRANZ, Yolanda, "La Inmigración latinoamericana en distintos contextos de recepción" Migraciones, Madrid, 1998, No 3, pp. 31-51.

JENSEN, Silvina, "La provincia flotante. El exilio argentino en Cataluña (1976-2006)", Casa América, Barcelona, 2008. 
JENSEN, Silvina y LASTRA, Soledad, "El problema de las escalas en el campo de estudio de los exilios políticos argentinos recientes”, en Avances del Cesor, Rosario, 2015, V. XII, pp. 97-119.

JIMÉNEZ, Cecilia, "Desclasamiento y reconversiones en las trayectorias de los migrantes argentinos de clases medias”, Tesis Doctoral, Universidad Complutense de Madrid, 2011.

KOGAN, Irena, "Last hired, first fired? The unemployment dynamics of male immigrants in Germany", European Sociological Review, Mannheim, 2004, V. 20, No 5, pp. 445-461.

KOSSOUDJI, Sherrie y COBB-CLARK, Deborah, "IRCA's impact on the occupational concentration and mobility of newly-legalized Mexican men”, en Journal of Population Economics, Maastricht, 2000, V. 13, No 1, pp. 81-98.

LAM, Kit-Chun y LIU, Pak-Wai, "Earnings divergence of immigrants", en Journal of Labor Economics, 2002, V. 20, No 1, pp. 86-104.

LONG,James E. “The effect of Americanization on earnings: Some evidence for women”. Journal of Political Economy, Chicago, 1980, V. 88, No 3, pp. 620-629.

MÁS, Fernando, “De Nüremberg a Madrid. Historia intima de un juicio”, Grijalbo, Buenos Aires, 1999.

MIRA DELLI-ZOTTI, Guillermo, “¿Sobrevivir o vivir en Madrid? Exiliados argentinos del '76”, en ESPINA BARRIO, Ángel (editor) Antropología en Castilla y León e Iberoamérica $V$. Emigración e integración cultural, Universidad de Salamanca, Salamanca, 2003, pp. 187-198.

MIRA DELLI-ZOTTI, Guillermo, ESTEBAN, Fernando, "El flujo que no cesa. Aproximación a las razones, cronología y perfil de los argentinos radicados en España (1975-2001)”, en Historia Actual OnLine, Cádiz, 2003, No 2, pp. 31-43

MINCER, Jacob, “Family migration decisions”, en Journal of Political Economy, Chicago, 1978, V. 86, No 5, pp. 749-773.

MINUJIN, Alberto, KESSLER, Gabriel, La nueva pobreza en la Argentina, Editorial Planeta, Buenos Aires, 1995.

MOULIER-BOUTANG, Yann, "L'avenir des migrations vers 1'Europe: changer de système migratoire et de paradigm”, en BRIBOSIA, Emmanuelle y REA, Andrea (directoras) Les nouvelles migrations. Un enjeu européen, Complex, Bruselas, 2002, pp. 75-91.

MULLAN, Brendan P., “The impact of social networks on the occupational status of migrants", en International Migration, Staten Island, 1989, V. 27, No 1, pp. 69-86.

ORGANIZACIÓN INTERNACIONAL PARA LAS MIGRACIONES (OIM), "Migrantes sudamericanos en España: panorama y políticas”, en Cuadernos Migratorios, Buenos Aires, 2001, No1.

OFICINA ECONÓMICA DEL PRESIDENTE, Inmigración y economía española: 1996-2006, Presidencia del Gobierno, Madrid, 2006. 
OSO, Laura, y VILLARES, María, "Mujeres inmigrantes latinoamericanas y empresariado étnico: dominicanas en Madrid, argentinas y venezolanas en Galicia", en Revista Galega de Economía, Santiago de Compostela, 2005, V. 14, №. 1 y 2, pp. 1-19

PATEL, Krishna y VELLA, Francis. "Immigrant networks and their implications for occupational choice and wages", en Review of Economics and Statistics, Cambridge, 2013, V. 95, No 4, pp. 1249-1277.

PIORE, Michael, "Notas para una teoría de la estratificación del mercado de trabajo", en TOHARIA, Luis, El mercado de trabajo: teorias y aplicaciones. Lecturas seleccionadas Alianza, Madrid, 1983, pp. 193-221.

PARELLA, Sonia, Mujer, inmigrante, trabajadora. La triple discriminación, Anthropos, Barcelona, 2003.

PIORE, Michael, Birds of Passage. Migrant Labour and industrial societies, Cambridge University Press, Cambridge, 1979.

PORTES, Alejandro, "Social Capital: Its Origins and Applications in Modern sociology", en Annual Review of Sociology, Palo Alto, 1998, 24, pp. 1-24.

PORTES, Alejandro y BÖRÖCZ, József, "Contemporary immigration: theoretical perspectives on its determinants and models of incorporation", en International Migration Review, Staten Island, 1989, V. 23, No 3, pp. 606-630. Disponible en: <http://www.jstor.org/stable/2546432>.

RAIJMAN, Rebeca; SEMYONOV, Moshe, "Gender, ethnicity, and immigration: Double disadvantage and triple disadvantage among recent immigrant women in the Israeli labor market", en Gender E Society, 1997, V. 11, No 1, pp. 108-125. REDSTONE AKRESH, Iliana, "Occupational mobility among legal immigrants to the United States", en International Migration Review, Staten Island, 2006, V. 40, No 4, pp. 854-884.

ROMERO, Luis A., La crisis argentina: una mirada al siglo XX, S.XXI, Buenos Aires, 2003.

SANTACREU, Oscar; BALDONI, Emiliana y ALBERT, Ma Carmen. "Deciding to move: migration projects in an integrating Europe", en RECCHI, Ettore y Favell, Adrian (editores) Pioneers of European integration: Citizenship and mobility in the EU, Edward Elgar Publishing Limited, Cheltenham, 2009, pp. 52-71.

SASSEN, Saskia, "La movilidad del trabajo y del capital. Un estudio de la corriente internacional de la inversión $y$ del trabajo", MTAS, Madrid, 1993.

SVAMPA, Maristella. "Las clases medias en Argentina", en Los que ganaron. La vida en los countries y barrios privados. Buenos Aires, Biblos, 2001, pp. 19-48

SVAMPA, Maristella, La sociedad excluyente. La Argentina bajo el signo del neoliberalismo, Taurus, Buenos Aires, 2005.

TORRADO, Susana, Estructura social de la Argentina (1945-1983), Ediciones de la Flor, Buenos Aires, 1994. 
VEIRA, Alberto, STANEK, Mikolaj y CACHÓN, Lorenzo, "Los determinantes de la concentración étnica en el mercado laboral español”, en Revista Internacional de Sociologia, Córdoba, 2011, V.69, Monográfico No 1, pp. 219-242.

VILLA, Paola, La estructuración de los mercados de trabajo, MTSS, Madrid, 1990.

VONO, Daniela y VIDAL, Elena, “The impact of informal networks on labour mobility: Immigrants' first job in Spain”, en Migration Letters, London, Reino Unido, 2012, V. 9, No 3, pp. 237-247.

YAMAUCHI, Futoshi y TANABE, Sakiko, "Nonmarket networks among migrants: Evidence from metropolitan Bangkok", en Journal of Population Economics, 2008, V. 21, No 3, pp. 649-664.

WEISS, Yoram, SAUER, Robert M., GOTLIBOVSKI, Menachem. "Immigration, search, and loss of skill”, en Journal of Labour Economics, Chicago, 2003, V. 21, No 3, 557-591. 